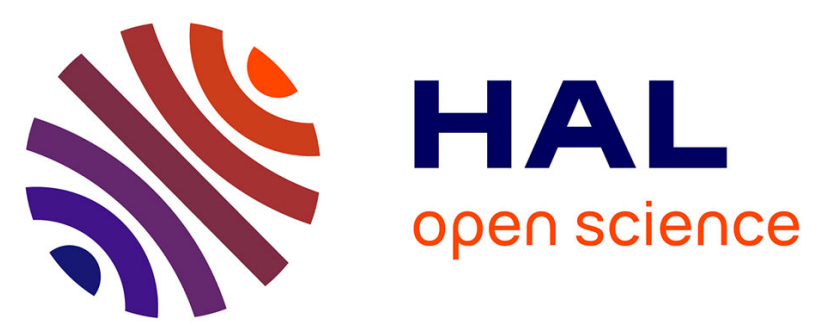

\title{
Small-Molecule Inhibitors of Cyclophilins Block Opening of the Mitochondrial Permeability Transition Pore and Protect Mice From Hepatic Ischemia/Reperfusion Injury
}

\author{
Mathieu Panel, Isaac Ruiz, Rozenn Brillet, Fouad Lafdil, Fatima
}

Teixeira-Clerc, Cong Trung Nguyen, Julien Caldéraro, Muriel Gelin, Fred

Allemand, Jean-François Guichou, et al.

\section{To cite this version:}

Mathieu Panel, Isaac Ruiz, Rozenn Brillet, Fouad Lafdil, Fatima Teixeira-Clerc, et al.. Small-Molecule Inhibitors of Cyclophilins Block Opening of the Mitochondrial Permeability Transition Pore and Protect Mice From Hepatic Ischemia/Reperfusion Injury. Gastroenterology, 2019, 157 (5), pp.1368-1382. 10.1053/j.gastro.2019.07.026 . hal-02391962

\section{HAL Id: hal-02391962 \\ https://hal.science/hal-02391962}

Submitted on 24 Nov 2020

HAL is a multi-disciplinary open access archive for the deposit and dissemination of scientific research documents, whether they are published or not. The documents may come from teaching and research institutions in France or abroad, or from public or private research centers.
L'archive ouverte pluridisciplinaire $\mathbf{H A L}$, est destinée au dépôt et à la diffusion de documents scientifiques de niveau recherche, publiés ou non, émanant des établissements d'enseignement et de recherche français ou étrangers, des laboratoires publics ou privés. 


\title{
Small-molecule inhibitors of cyclophilins block opening of the mitochondrial permeability transition pore and protect mice from hepatic ischemia-reperfusion injury
}

Mathieu Panel, ${ }^{1,2 *}$ Isaac Ruiz, ${ }^{3 *}$ Rozenn Brillet, ${ }^{3}$ Fouad Lafdil, ${ }^{3,4}$ Fatima Teixeira-Clerc, ${ }^{3}$ Cong Trung Nguyen, ${ }^{3,5}$ Julien Calderaro, ${ }^{3,5}$ Muriel Gelin, ${ }^{6}$ Fred Allemand, ${ }^{6}$ Jean-François Guichou, ${ }^{6}$ Bijan Ghaleh, ${ }^{1,2}$ Abdelhakim Ahmed-Belkacem, ${ }^{3 \#}$ Didier Morin, ${ }^{1,2 \#}$ and Jean-Michel Pawlotsky,7\#

\section{${ }^{1}$ INSERM U955, Team 3, Créteil, France; ${ }^{2}$ Université Paris-Est, UMR S955, DHU A-TVB,} UPEC, Créteil, France; ${ }^{3}$ INSERM U955, Team "Pathophysiology and Therapy of Chronic Viral Hepatitis and Related Cancers", Créteil, France; ${ }^{4}$ Institut Universitaire de France (IUF), Paris, France; ${ }^{5}$ Department of Pathology, Hôpital Henri Mondor, Université Paris-Est, Créteil,

France; ${ }^{6}$ Centre de Biochimie Structurale (CBS), INSERM U1054, CNRS UMR5048,

Université de Montpellier, Montpellier, France; ${ }^{7}$ National Reference Center for Viral Hepatitis B, C and Delta, Department of Virology, Hôpital Henri Mondor, Université ParisEst, Créteil, France

\begin{abstract}
Background \& Aims: Hepatic ischemia-reperfusion injury is a complication of liver surgery that involves mitochondrial dysfunction resulting from mitochondrial permeability transition pore (mPTP) opening. Cyclophilin D (PPIF or CypD) is a peptidyl-prolyl cis-trans isomerase that regulates mPTP opening in the inner mitochondrial membrane. We investigated whether and how recently created small-molecule inhibitors of CypD prevent opening of the mPTP in hepatocytes and the resulting effects in cell models and livers of mice undergoing ischemia-reperfusion injury.

Methods: We measured the activity of 9 small-molecule inhibitors of Cyps in an assay of CypD activity. The effects of the small-molecule CypD inhibitors or vehicle on mPTP opening were assessed by measuring mitochondrial swelling and calcium retention in isolated liver mitochondria from C57BL/6J (wild-type) and Ppif ${ }^{-}$(CypD knock-out) mice, and in primary mouse and human hepatocytes by fluorescence microscopy. We induced ischemiareperfusion injury in livers of mice given a small-molecule CypD inhibitor or vehicle before and during reperfusion, and collected samples of blood and liver for histologic analysis.

Results: The compounds inhibited peptidyl-prolyl isomerase activity ( $\mathrm{IC}_{50}$ values, 0.2 to $16.2 \mu \mathrm{M})$ and, as a result, calcium-induced mitochondrial swelling, by preventing mPTP opening ( $\mathrm{IC}_{50}$ values, 1.4 to $132 \mu \mathrm{M}$ ) in a concentration-dependent manner. The most potent inhibitor (C31) bound CypD with high affinity and inhibited swelling in mitochondria from livers of wild-type and $\mathrm{Ppif}^{/-}$mice (indicating an additional, CypD-independent effect on mPTP opening) and in primary human and mouse hepatocytes. Administration of C31 in mice with ischemia-reperfusion injury before and during reperfusion restored hepatic calcium retention capacity and oxidative phosphorylation parameters and reduced liver damage compared with vehicle.
\end{abstract}

KEY WORDS: drug, mitochondrial swelling, PPIase activity, mouse model.

doi: 10.1053/j.gastro.2019.07.026. 
Small-molecule inhibitors of cyclophilins block opening of the mitochondrial permeability transition pore and protect mice from hepatic ischemia-reperfusion injury

Mathieu Panel, ${ }^{1,2 *}$ Isaac Ruiz, ${ }^{3 *}$ Rozenn Brillet, ${ }^{3}$ Fouad Lafdil, ${ }^{3,4}$ Fatima Teixeira-Clerc, ${ }^{3}$ Cong Trung Nguyen, ${ }^{3,5}$ Julien Calderaro, ${ }^{3,5}$ Muriel Gelin, ${ }^{6}$ Fred Allemand, ${ }^{6}$ Jean-François Guichou, ${ }^{6}$ Bijan Ghaleh, ${ }^{1,2}$ Abdelhakim Ahmed-Belkacem, ${ }^{3 \#}$ Didier Morin, ${ }^{1,2 \#}$ and Jean-Michel Pawlotsky 3 ,7\#

*Authors share co-first authorship

\#Authors share co-senior authorship.

${ }^{1}$ INSERM U955, Team 3, Créteil, France; ${ }^{2}$ Université Paris-Est, UMR S955, DHU A-TVB, UPEC, Créteil, France; ${ }^{3}$ INSERM U955, Team “Pathophysiology and Therapy of Chronic Viral Hepatitis and Related Cancers“, Créteil, France; ${ }^{4}$ Institut Universitaire de France (IUF), Paris, France; ${ }^{5}$ Department of Pathology, Hôpital Henri Mondor, Université Paris-Est, Créteil, France; ${ }^{6}$ Centre de Biochimie Structurale (CBS), INSERM U1054, CNRS UMR5048, Université de Montpellier, Montpellier, France; ${ }^{7}$ National Reference Center for Viral Hepatitis B, C and Delta, Department of Virology, Hôpital Henri Mondor, Université ParisEst, Créteil, France

\section{Corresponding authors:}

- Jean-Michel Pawlotsky :Department of Virology, Hôpital Henri Mondor, 51 avenue du Maréchal de Lattre de Tassigny, 94010 Créteil, France. E-mail : jean-michel.pawlotsky@aphp.fr 
- Didier Morin : INSERM U955, équipe 3, Faculté de Médecine, 8 rue du général Sarrail, 94000, Créteil, France.

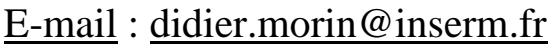

Grant support: Mathieu Panel was supported by a grant from the French Ministry for Higher Education and Research (Grant \#2014-140). Isaac Ruiz was supported by grants from the National Agency for Research on AIDS and Viral Hepatitis (ANRS) and the Mexican National Council of Science and Technology (CONACYT).

Conflict of interest disclosures: Inserm Transfert is the owner of patent EP 09306294.1 covering the family of cyclophilin inhibitors described, for which Abdelhakim AhmedBelkacem, Jean-François Guichou and Jean-Michel Pawlotsky are inventors. All other authors declare no competing financial interests.

Acknowledgement: The authors are grateful to Drs Joseph Benga and Stéphane Moutereau from the Department of Biochemistry at Henri Mondor hospital for ALT and AST level measurements and to Prof. René Tolba and Dr Zoltan Czigany from the Aachen University Hospital, Germany, for helpful discussions regarding the in vivo ischemia-reperfusion injury model.

\section{Authors contributions:}

- Mathieu Panel: acquisition, analysis and interpretation of data; drafting of the manuscript; statistical analysis.

- Isaac Ruiz: contribution to the experimental design of murine ischemia-reperfusion experiments; acquisition, analysis and interpretation of data.

- Rozenn Brillet: acquisition, analysis and interpretation of data. 
- Fouad Lafdil: contribution to the experimental design of murine ischemia-reperfusion experiments; revision of the manuscript.

- Fatima Teixeira-Clerc: contribution to the experimental design of murine ischemiareperfusion experiments; revision of the manuscript.

104 - Cong Trung Nguyen: acquisition, analysis and interpretation of histological data.

- Julien Calderaro: acquisition, analysis and interpretation of histological data.

- Muriel Gelin: acquisition, analysis and interpretation of chemical data.

- Fred Allemand: acquisition, analysis and interpretation of chemical data.

- Jean-François Guichou: compound design and synthesis; generation of structural data; revision of the manuscript.

- Abdelhakim Ahmed-Belkacem: study supervision; acquisition, analysis and interpretation of data; drafting of the manuscript.

- Didier Morin: study supervision; analysis and interpretation of data; drafting of the manuscript.

- Jean-Michel Pawlotsky: study supervision; analysis and interpretation of data; critical revision of the manuscript for important intellectual content. 
119

120

121

122

123

124

125

126

127

\section{Abstract}

Background \& Aims: Hepatic ischemia-reperfusion injury is a complication of liver surgery that involves mitochondrial dysfunction resulting from mitochondrial permeability transition pore (mPTP) opening. Cyclophilin D (PPIF or CypD) is a peptidyl-prolyl cis-trans isomerase that regulates $\mathrm{mPTP}$ opening in the inner mitochondrial membrane. We investigated whether and how recently created small-molecule inhibitors of CypD prevent opening of the mPTP in hepatocytes and the resulting effects in cell models and livers of mice undergoing ischemia-reperfusion injury.

Methods: We measured the activity of 9 small-molecule inhibitors of Cyps in an assay of CypD activity. The effects of the small-molecule CypD inhibitors or vehicle on mPTP opening were assessed by measuring mitochondrial swelling and calcium retention in isolated liver mitochondria from C57BL/6J (wild-type) and Ppif ${ }^{-}$(CypD knock-out) mice, and in primary mouse and human hepatocytes by fluorescence microscopy. We induced ischemiareperfusion injury in livers of mice given a small-molecule CypD inhibitor or vehicle before and during reperfusion, and collected samples of blood and liver for histologic analysis.

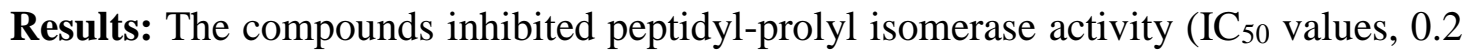
to $16.2 \mu \mathrm{M})$ and, as a result, calcium-induced mitochondrial swelling, by preventing mPTP opening ( $\mathrm{IC}_{50}$ values, 1.4 to $132 \mu \mathrm{M}$ ) in a concentration-dependent manner. The most potent inhibitor (C31) bound CypD with high affinity and inhibited swelling in mitochondria from livers of wild-type and $\mathrm{Ppif}^{/-}$mice (indicating an additional, CypD-independent effect on mPTP opening) and in primary human and mouse hepatocytes. Administration of C31 in mice with ischemia-reperfusion injury before and during reperfusion restored hepatic calcium retention capacity and oxidative phosphorylation parameters and reduced liver damage compared with vehicle. 
Conclusions: Recently created small-molecule inhibitors of CypD reduced calcium-

145 induced swelling in mitochondria from mouse and human liver tissues. Administration of these

146 compounds to mice during ischemia-reperfusion restored hepatic calcium retention capacity

147 and oxidative phosphorylation parameters and reduced liver damage. These compounds might

148 be developed to protect patients from ischemia-reperfusion injury after liver surgery or for

149 other hepatic or non-hepatic disorders related to abnormal mPTP opening.

150

151 KEY WORDS: drug, mitochondrial swelling, PPIase activity, mouse model. 


\section{Introduction}

Hepatic ischemia-reperfusion injury is a major complication of liver surgery, including liver resection, liver transplantation, and trauma surgery ${ }^{1}$. Ischemia results from the interruption of the blood flow that perturbs the cellular metabolism as a result of oxygen shortage. Reperfusion restores the blood flow, oxygen delivery and tissue $\mathrm{pH}$, thereby exacerbating cellular damage initiated during hypoxia or anoxia ${ }^{2,3}$. Hepatic ischemia-reperfusion injury is an important cause of liver dysfunction or functional failure post-liver surgery, which affects perioperative morbidity, mortality and recovery ${ }^{2,3}$. There are two types of ischemia: the most frequent form, warm ischemia, is observed when vascular occlusion occurs during hepatic resection surgery or during exposure to low-flow incidences such as trauma, hemorrhagic shock, cardiac arrest or hepatic sinusoidal obstruction syndrome; cold ischemia is observed exclusively during orthotopic liver transplantation, when the graft is subjected to hypothermic preservation before warm reperfusion ${ }^{3}$. Their mechanisms and main target cells differ. Hepatocytes are a major target of warm ischemia-reperfusion injury through anoxia, nutrition depletion and cytosolic acidosis ${ }^{3}$. In particular, sodium, chloride and calcium homeostasis are significantly altered.

Mitochondrial dysfunction plays a major role in hepatic ischemia-reperfusion injury ${ }^{3}$. Indeed, ischemia-reperfusion triggers the mitochondrial permeability transition, characterized by an increase in the permeability of the inner mitochondrial membrane mediated by the opening of a channel, called the "mitochondrial permeability transition pore" $(\mathrm{mPTP})^{4}$. Once mitochondrial permeability transition initiates, solutes with a molecular mass of up to $1.5 \mathrm{kDa}$ diffuse across the mitochondrial inner membrane, inducing mitochondrial depolarization, uncoupling and swelling, which in turn induce ATP depletion and necrotic (and, to a lesser extent, apoptotic) cell death ${ }^{3}$. During hepatic ischemia-reperfusion, mPTP opening is triggered by calcium-mediated mitochondrial reactive oxygen species formation. The same phenomenon 
has been reported to play an important role during ischemia-reperfusion affecting other organs (including heart and brain) ${ }^{5-8}$, neurodegenerative diseases ${ }^{9}$ and drug-induced liver injury ${ }^{10}$.

Therapies avoiding the consequences of hepatic ischemia-reperfusion injury remain limited. Given the key role of mPTP opening in ischemia-reperfusion injury, pharmacological inhibition of mPTP opening is a promising target for new therapies. However, the molecular structure and functioning of mPTP remain largely unknown. Several proteins have been suggested to be involved in the structure of mPTP, including ATP synthase, the adenine nucleotide translocase, a phosphate carrier, and cyclophilin $\mathrm{D}(\mathrm{CypD})^{11}$. Cyclophilins are peptidyl prolyl cis/trans isomerases (PPIases) that catalyze the interconversion of the two energetically preferred conformers (cis and trans) of the planar peptide bond preceding an internal proline residue. Seventeen human cyclophilins have been identified, but the function of most of them is unknown ${ }^{12,13}$. CypD is located within the mitochondrial matrix where it acts as a key component and regulator of the $\mathrm{MPTP}$; $\mathrm{mPTP}$ formation appears to be catalyzed or stabilized by CypD through lowering of its calcium threshold ${ }^{14}$. Thus, CypD represents an attractive target for mPTP opening inhibition and cellular protection in the context of hepatic ischemia-reperfusion injury.

Cyclosporine A (CsA) and its derivatives (together with sanglifehrin A) are known macromolecular CypD ligands. They have been shown to efficiently desensitize mPTP opening in various cellular and in vivo models ${ }^{15-18}$. However, CsA is a potent immunosuppressor and its non-immunosuppressive derivatives suffer from many disadvantages, including their size, complex multistep synthesis, cell toxicity and lack of chemical plasticity. Thus, potent cyclophilin inhibitors unrelated to CsA or sanglifehrin A and lacking the disadvantages of nonimmunosuppressive macromolecular CsA derivatives are needed.

By means of a fragment-based drug discovery approach based on X-ray crystallography and nuclear magnetic resonance, we generated a new family of nonpeptidic small-molecule cyclophilin inhibitors (SMCypIs) unrelated to CsA or sanglifehrin A, with potent inhibitory 
activity against $\mathrm{CypA}, \mathrm{CypB}$ and $\mathrm{CypD}^{19}$. These compounds lack cellular toxicity and immunosuppressive activity and bear druggable pharmacological properties ${ }^{19}$. In this study, we assessed the ability of the new SMCypIs to inhibit liver mitochondria mPTP opening through CypD inhibition, studied their mechanisms of inhibition, and evaluated in vivo their protective properties in the context of experimental hepatic ischemia-reperfusion injury.

\section{Materials and Methods}

\section{Drugs and cells}

Unless specified, all reagents were purchased from Sigma-Aldrich (Saint-Quentin Fallavier, France). Calcein AM (C3100MP) and calcium Green 5N (C3737) were obtained from Invitrogen (Cergy-Pontoise, France). SMCypIs were synthesized as previously described ${ }^{19}$. Primary human hepatocytes were obtained from Biopredict International (Saint-Grégoire, France).

\section{Animals}

Male C57BL/6J mice (8 to 10-week-old) were purchased from Janvier (Le Genest-StIsle, France). Ppif $^{/-}$mice (i.e., CypD knock-out mice) were obtained from Jackson Laboratories (Bar Harbor, Maine). Animals were co-housed in an air-conditioned room with a 12-h lightdark cycle and received standard rodent chow and drinking water ad libitum. All animal procedures in this study were in strict accordance with the Directives of the European Parliament (2010/63/EU-848 EEC) and approved by the Animal Ethics Committee ANSES/ENVA/Université Paris-Est Créteil (approval numbers 09/12/14-02 and 11/10/16-01). 


\section{Molecular modeling and docking of C31 into CypD}

Molecular modeling and docking experiments were performed by means of the @ TOME-2 server ${ }^{20}$. In each structural model, the boundaries of the active site were deduced from the vicinity of co-crystallized ligands (compounds C30, C27, and C24 selected as templates, with PDB accession numbers 4J5C, 4J5B and 4J5E, respectively) using @ TOME-2 comparative option. They were used to guide docking in automatically computed models. The ligand files were generated with MarvinSketch 6.2.2 for SMILES and Frog2 server for mol2 $2^{21}$. The Figure was generated by means of Pymol.

\section{CypD PPIase enzyme assay}

Inhibition of CypD PPIase activity was measured at $20^{\circ} \mathrm{C}$ by means of the standard chymotrypsin coupled assay. The assay buffer (25 mM HEPES, $100 \mathrm{mM} \mathrm{NaCl}, \mathrm{pH} 7.8$ ) and purified CypD (1900 nM stock solution) were pre-cooled at $4{ }^{\circ} \mathrm{C}$ in the presence of serial dilutions of the SMCypIs. Five $\mu \mathrm{L}$ of $50 \mathrm{mg} / \mathrm{mL}$ chymotrypsin in $1 \mathrm{mM} \mathrm{HCl}$ was added. The reaction was initiated by adding $20 \mu \mathrm{L}$ of $3.2 \mathrm{mM}$ peptide substrate (N-succinyl-Ala-Ala-CisPro-Phe-p-nitroanilide). P-nitroanilide absorbance was measured at $390 \mathrm{~nm}$ for $1 \mathrm{~min}$. CsA was used as a positive control of PPIase inhibition. The percent inhibition of CypD PPIase activity was calculated from the slopes and the $\mathrm{IC}_{50 \mathrm{~s}}$ were determined from percent inhibition curves using Sigmaplot software.

\section{Isolation of liver mitochondria}

Mouse livers were scissor-minced and homogenized on ice in a buffer $(220 \mathrm{mM}$ mannitol, $70 \mathrm{mM}$ sucrose, $10 \mathrm{mM}$ HEPES, $4 \mathrm{mM}$ EGTA, pH 7.4 at $4^{\circ} \mathrm{C}$ ) using a Potter- 
Elvehjem glass homogenizer in a final volume of $10 \mathrm{~mL}$. The homogenate was centrifuged at $1000 \mathrm{~g}$ for $5 \mathrm{~min}$ at $4{ }^{\circ} \mathrm{C}$. The supernatant was centrifuged at $10,000 \mathrm{~g}$ for $10 \mathrm{~min}$ at $4^{\circ} \mathrm{C}$. The mitochondrial pellet was resuspended in $600 \mu \mathrm{l}$ of homogenization buffer without EGTA and protein concentration was determined.

\section{Measurement of mitochondrial oxygen consumption}

Oxygen consumption of isolated mitochondria was measured with a Clark-type electrode fitted to a water-jacketed reaction chamber (Hansatech, Cergy, France). Mitochondria $\left(1 \mathrm{mg}\right.$ protein $/ \mathrm{mL}$ ) were incubated at $30^{\circ} \mathrm{C}$ in a respiration buffer containing $100 \mathrm{mM} \mathrm{KCl}, 50$ $\mathrm{mM}$ sucrose, $10 \mathrm{mM}$ HEPES and $5 \mathrm{mM} \mathrm{KH}_{2} \mathrm{PO}_{4}$ at $\mathrm{pH}$ 7.4. Respiration was initiated by the addition of glutamate/malate ( $5 \mathrm{mM}$ each). After $1 \mathrm{~min}$, ATP synthesis was induced by the addition of $1 \mathrm{mM}$ ADP (state 3 respiration rate), and $1 \mu \mathrm{M}$ carboxyatractyloside was added to measure the substrate-dependent respiration rate (state 4). The respiratory control ratio (state 3/state 4) was then calculated.

\section{Mitochondrial swelling assays}

Mitochondrial swelling was assessed by measuring the change in absorbance at $540 \mathrm{~nm}$ $\left(\mathrm{A}_{540}\right)$ using a Jasco V-530 spectrophotometer equipped with magnetic stirring and thermostatic control. Mitochondria $(0.5 \mathrm{mg} / \mathrm{mL})$ energized with pyruvate/malate (5 mM each) were incubated $30 \mathrm{sec}$ in the respiration buffer before the induction of swelling with $10 \mathrm{mM}$ phosphate, $100 \mu \mathrm{M}$ atractyloside or $40 \mu \mathrm{M}$ tert-butyl hydroperoxide in the presence of $50 \mu \mathrm{M}$ of $\mathrm{CaCl}_{2}$.

In de-energized conditions, mitochondria $(0.5 \mathrm{mg} / \mathrm{mL})$ were incubated $1 \mathrm{~min}$ at $30^{\circ} \mathrm{C}$ in a swelling buffer containing $100 \mathrm{mM} \mathrm{KCl,} 50 \mathrm{mM}$ sucrose, $10 \mathrm{mM}$ HEPES, $5 \mathrm{mM} \mathrm{KH}_{2} \mathrm{PO}_{4}, 1$ 
$\mu \mathrm{M}$ rotenone, and $1 \mu \mathrm{M}$ antimycin at $\mathrm{pH}$ 7.4. Then, $50 \mu \mathrm{M}$ or $100 \mu \mathrm{M} \mathrm{CaCl}_{2}$ was added 1 min before swelling induction with $1 \mu \mathrm{M}$ of $\mathrm{A} 23187$ or $10 \mu \mathrm{M}$ phenylarsine oxide, respectively. In both conditions, SMCypIs or CsA were introduced at the beginning of the incubation period.

\section{Measurement of the calcium retention capacity of isolated mitochondria}

Mitochondria were loaded with increasing concentrations of calcium until mPTP opening and fast calcium release occurred ${ }^{22}$. Liver mitochondria $(1 \mathrm{mg} / \mathrm{mL})$ energized with 5 $\mathrm{mM}$ glutamate/malate were incubated in the respiration buffer supplemented with $1 \mu \mathrm{M}$ Calcium Green-5N fluorescent probe. The calcium concentration in the extra-mitochondrial medium was monitored by means of a Jasco FP-6300 spectrofluorimeter (Jasco, Bouguenais, France) at excitation and emission wavelengths of 506 and $532 \mathrm{~nm}$, respectively. The calcium signal was calibrated by addition of known calcium amounts to the medium.

\section{Isolation of primary mouse hepatocytes}

Murine hepatocytes from C57BL6 animals were isolated by portal vein perfusion of collagenase $^{23}$. Freshly isolated hepatocytes were cultured in DMEM supplemented with $10 \%$ fetal calf serum, $10 \mathrm{U} / \mathrm{mL}$ penicillin, $10 \mu \mathrm{g} / \mathrm{mL}$ streptomycin, $10 \mu \mathrm{g} / \mathrm{mL}$ insulin, $5.5 \mu \mathrm{g} / \mathrm{mL}$ transferrin and $5 \mathrm{ng} / \mathrm{mL}$ sodium selenite. Four hours post-perfusion, the medium was removed and fresh medium, supplemented with $0.1 \mu \mathrm{M}$ dexamethasone and $50 \mathrm{ng} / \mathrm{mL}$ epidermal growth factor, was added.

\section{Measurement of MPTP opening in primary mouse and human hepatocytes}


mPTP opening was assessed in primary mouse and human hepatocytes by inducing mitochondrial localization of calcein fluorescence ${ }^{24}$. Cells were loaded with $2 \mathrm{mM} \mathrm{CoCl}_{2}$ at $30^{\circ} \mathrm{C}$ in $1 \mathrm{~mL}$ of Tyrode's solution $\left(\mathrm{NaCl} 130 \mathrm{mM}, \mathrm{KCl} 5 \mathrm{mM}\right.$, HEPES $10 \mathrm{mM}, \mathrm{MgCl}_{2} 1 \mathrm{mM}$, $\mathrm{CaCl}_{2} 1.8 \mathrm{mM}$, pH 7.4 at $37^{\circ} \mathrm{C}$ ) for $30 \mathrm{~min}$. After $10 \mathrm{~min}$, the cells were supplemented with 1 $\mu \mathrm{M}$ calcein acetoxymethyl ester, washed free of calcein and $\mathrm{CoCl}_{2}$, placed in a thermostated chamber and incubated in Tyrode's solution. After $5 \mathrm{~min}, 2 \mu \mathrm{M}$ of calcium ionophore A23187 was added to induce mPTP opening ${ }^{25}$. When specified, either C31 or CsA was added at the beginning of the incubation period.

Cells were imaged with an Olympus IX-81 motorized inverted microscope equipped with a mercury lamp as the light source for epifluorescence illumination, combined with a 12bit cooled Hamamatsu ORCA-ER camera; 460-490 nm excitation and $510 \mathrm{~nm}$ emission filters were used for detection of calcein fluorescence. Images were acquired every min for $30 \mathrm{~min}$ after $30 \mathrm{~ms}$ of illumination per image using a digital epifluorescence imaging software (Cell M, Olympus, Rungis, France). Fluorescence from each cell in each region of interest $\left(\approx 80 \mu \mathrm{m}^{2}\right)$ was measured and background fluorescence was subtracted. Average fluorescence changes per field were calculated. Fluorescence was normalized to the maximal fluorescence value.

\section{Ex vivo assessment of mPTP opening in mice treated with C31}

The mice were anesthetized by intraperitoneal injection of pentobarbital sodium ( 80 $\mathrm{mg} / \mathrm{kg})$. Increasing doses of C31 $(10,20,50$ and $150 \mathrm{mg} / \mathrm{kg})$ or vehicle were randomly administered as a 3-min infusion through the jugular vein. Two min after the end of the infusion, the livers were excised and mitochondria were isolated to measure their capacity to retain calcium, as a marker of the ability of $\mathrm{C} 31$ to inhibit mPTP opening in vivo. 
In vivo assessment of the mito- and hepatoprotective effect of SMCypIs in the context of hepatic ischemia-reperfusion

The mice were anesthetized by intraperitoneal injection of pentobarbital sodium ( 80 $\mathrm{mg} / \mathrm{kg}$ ), intubated and mechanically ventilated. After section of the liver ligaments, hepatic normothermic ischemia of segments I to $\mathrm{V}$ was induced for $60 \mathrm{~min}$ by hilum clamping of the hepatic pedicle. Reperfusion occurred at removal of the clamp. One min before reperfusion, C31 or the vehicle was infused through the jugular vein and the infusion was maintained for the first 8 min of reperfusion.

Two sets of experiments were conducted. In the first set, livers were excised after 10 min of reperfusion and mitochondria were isolated for ex vivo experiments. In the second set, reperfusion was stopped after $60 \mathrm{~min} ; 500 \mu \mathrm{L}$ of blood was collected to measure alanine aminotransferase (ALT) and aspartate aminotransferase (AST) levels, while livers were excised, formalin-fixed, paraffin-embedded and liver sections were stained with hematoxylin and eosin. "Hepatocyte clarification", a histological feature of severe hepatocellular damage characterized by hepatocyte ballooning degeneration, was measured on whole sections of mice livers. Histological slides were scanned at an x20 magnification by means of an Aperio Slide Scanner (Leica Biosystems, Shanghai, China). Slides in ".svs" format were analyzed by means of Qupath open source software ${ }^{26}$. Areas with hepatocyte ballooning were then manually annotated independently by two pathologists specialized in liver diseases.

\section{Statistical analysis}

Results are expressed as mean \pm SEM of at least 5 independent experiments. Statistical analysis was performed using one-way ANOVA followed by the Scheffé post-hoc test. Differences were considered significant for $p<0.05$. 
Results

\section{SMCypIs bind CypD and inhibit its PPIase activity}

We recently created a new family of small-molecule, nonpeptidic cyclophilin inhibitors $(\text { SMCypI })^{19}$. As shown in Supplementary Table 1 and Supplementary Figure 1, 9 members of the new SMCypI family, including compound C22, compounds C23 to C27 and compounds C29 to C31 (which all resulted from structure-guided chemical optimization of C22), inhibited CypD PPIase activity in an enzyme assay, with $\mathrm{IC}_{50}$ values ranging from 0.2 to $16.2 \mu \mathrm{M}$. The most potent PPIase inhibitor was $\mathrm{C} 31$, with an $\mathrm{IC}_{50}$ of $0.2 \pm 0.08 \mu \mathrm{M}$. Molecular modeling and docking experiments revealed that $\mathrm{C} 31$ binds both the catalytic site and the gatekeeper pocket of CypD (Figure 1A). activity in isolated mouse liver mitochondria

As shown in Figure 1B, $1 \mu \mathrm{M}$ CsA, used as a positive control of CypD inhibition, fully inhibited calcium-induced swelling of energized isolated mouse liver mitochondria. The 9 SMCypIs also inhibited mitochondrial swelling in a concentration-dependent manner (Supplementary Table 1). The most effective compound was C31, with an $\mathrm{IC}_{50}$ of $1.4 \pm 0.2 \mu \mathrm{M}$ (Figure 1B). SMCypI inhibition of calcium-induced mitochondrial swelling strongly correlated with SMCypI inhibition of CypD PPIase activity ( $\mathrm{r}=0.8535$; $\mathrm{p}<0.004$; Figure $1 \mathrm{C})$. mitochondrial swelling as a result of CypD PPIase activity inhibition by binding to both the 
PPIase catalytic site and the gatekeeper pocket of CypD. C31, the most potent SMCypI, was selected for subsequent experiments.

\section{SMCypI inhibition of mitochondrial swelling is due to the inhibition of mPTP opening}

To assess whether mitochondrial swelling inhibition by SMCypIs is related to the inhibition of mPTP opening, swelling was induced by different known mPTP opening inducers. As shown in Figures 2A and 2B, C31 inhibited mitochondrial swelling induced by both the prooxidant drug tert-butyl hydroperoxide and carboxyatractyloside, a ligand of adenine nucleotide translocase, in energized conditions.

Because mitochondrial parameters, such as the membrane potential or the ATP/ADP ratio, can modulate $\mathrm{mPTP}$ opening under energized conditions, thereby influencing the observed effect of the inhibitors, the inhibitory effect of C31 was analyzed under de-energized conditions, i.e., in the absence of substrate. Mitochondria were incubated in the presence of rotenone and antimycin to fully block the respiratory chain and supplemented with the calcium ionophore A23187 or the cross-linking thiol agent phenylarsine oxide in the presence of calcium. In these conditions (Figure 2C and 2D, respectively), mitochondrial swelling was slower than under energized conditions, but still inhibited by SMCypI C31.

These results indicate that SMCypI compound C31 inhibits mitochondrial swelling by inhibiting mPTP opening.

SMCypI compound C31 inhibits MPTP opening in both primary mouse and human hepatocytes

Primary mouse hepatocytes were loaded with calcein in the presence of $\mathrm{CoCl}_{2}$ and mPTP opening was induced by the addition of $1 \mu \mathrm{M}$ A23187 (Figure 3A). As shown in Figure 
3B, CsA inhibited the drop in calcein fluorescence associated with mPTP opening by only $55.4 \pm 0.8 \%$ at $30 \mathrm{~min}$ at the most effective concentration of $2 \mu \mathrm{M} . \mathrm{C} 31$ inhibited the drop in calcein fluorescence in a concentration-dependent manner, a result indicating that C31 inhibits mPTP opening in living mouse liver cells (Figure 3B). The inhibitory effect of C31 was stronger than that of $2 \mu \mathrm{M}$ CsA, with full inhibition of mPTP opening obtained with $100 \mu \mathrm{M}$ of $\mathrm{C} 31$.

The same experiments were performed with primary human hepatocytes (Figure 3C). CsA inhibited mPTP opening by approximately $50 \%$ at the most effective concentration of 0.2 $\mu \mathrm{M}$. C31 inhibited mPTP opening in a concentration-dependent manner in primary human hepatocytes, with full inhibition achieved at the concentration of $100 \mu \mathrm{M}$ (Figure 3C). This indicated that results obtained in mouse liver cells are representative of the human liver situation.

\section{SMCypI compound C31 is more potent than CsA at increasing mitochondrial calcium} retention capacity in isolated mouse mitochondria

The calcium retention capacity of isolated mouse mitochondria (i.e., the maximal calcium load achievable in the presence of a drug before mPTP opens) was measured, as previously described ${ }^{22}$. As shown in Figures $4 \mathrm{~A}$ and $4 \mathrm{~B}, 6 \pm 1$ additions of $20 \mu \mathrm{M}$ calcium, corresponding to $109 \pm 15 \mathrm{nmol} / \mathrm{mg}$ of mitochondrial proteins, were required to induce $\mathrm{mPTP}$ opening in the absence of CypD inhibitors. One $\mu \mathrm{M}$ of CsA increased the mitochondrial calcium retention capacity by 3 -fold $(337 \pm 47 \mathrm{nmol} / \mathrm{mg}$ of mitochondrial proteins). $\mathrm{C} 31$ at concentrations up to $100 \mu \mathrm{M}$ also increased the mitochondrial calcium retention capacity. The maximum effect of $\mathrm{C} 31$ was markedly greater than that of CsA $(550 \pm 37 \mathrm{nmol} / \mathrm{mg}$ of mitochondrial proteins; Figures 4A and 4B). C31 also more potently increased mitochondrial calcium retention capacity than CsA when the respiratory chain was fed with succinate in the 
presence of rotenone, indicating that this effect is independent from activated respiratory complex I or II (data not shown).

Maximum mitochondrial calcium retention capacities of the same order were achieved in the presence of $10 \mu \mathrm{M}$ of $\mathrm{C} 31$ and $1 \mu \mathrm{M}$ of CsA, respectively. At these concentrations, that correspond to approximately 10 times the respective compound $\mathrm{IC}_{50}$ values, $\mathrm{CypD}$ saturation is achieved. The combination of both $\mathrm{C} 31$ and CsA at the same concentrations did not result in any additive effect (Figure 4B), a result in keeping with them sharing CypD as a target.

Together, these results indicate that SMCypI compound C31 inhibits mPTP opening at least partly through CypD inhibition, and that this inhibition is more potent than that achieved by CsA.

The additional effectiveness of C31 as compared to CsA in inhibiting mPTP opening is unrelated to CypD inhibition

The mitochondrial calcium retention capacity in the presence of $100 \mu \mathrm{M}$ C31 was not modified by the addition of $1 \mu \mathrm{M}$ CsA (Figure 4B). This suggests that the greater capacity of C31 to inhibit mPTP opening as compared to CsA is unrelated to CypD inhibition. To verify this hypothesis, the effects of C31 and CsA were assessed on liver mitochondria isolated from Ppif $^{/-}$mice, which have been knocked-out for CypD, in comparison with wild-type animals. As previously shown ${ }^{27}$, the mitochondrial calcium retention capacity of Ppif ${ }^{/-}$mice was approximately 4-fold greater than that of wild-type animals (Figure 5A and 5B). CsA $1 \mu \mathrm{M}$ had no effect on Ppif $^{/-}$mice mitochondrial calcium retention capacity. In contrast, C31 increased the calcium retention capacity by approximately 1.5 -fold at concentrations $>20 \mu \mathrm{M}$ in mitochondria isolated from Ppif ${ }^{/-}$mice livers (Figure 5A and 5B). The C31 concentrations at which this effect was observed were higher than those required to inhibit CypD PPIase activity and mitochondrial swelling and to enhance calcium retention capacity in wild-type mouse 
mitochondria (see Figures 1 and 3 for comparison). In addition, C31 inhibited swelling induced by very high calcium concentrations in mitochondria isolated from $\mathrm{Ppif}^{/-}$mice (Figure 5C).

These results indicate that, in addition to blocking CypD, C31 interacts with another target at the mPTP level and that this interaction increases $\mathrm{C} 31$ blocking effectiveness on $\mathrm{mPTP}$ opening as compared to exclusive CypD inhibitors, such as CsA.

The additional effectiveness of C31 as compared to CsA in inhibiting mPTP opening is related to the chemical structure of its phenyl moiety

Comparison of the chemical structures of the SMCypIs (Supplementary Figure 1) suggested that the phenyl moiety of C31 is involved in this additional effect: indeed, SMCypI compound $\mathrm{C} 34$, that lacks the aromaticity of the cycle, lost the additional effect of C31 over CypD inhibition in isolated $\mathrm{Ppif}^{-f_{-}^{-}}$mouse liver mitochondria shown in Figure 5D.

The additional effectiveness of C31 as compared to CsA in inhibiting mPTP opening is unrelated to a ubiquinone-like effect or an interaction with the mitochondrial respiratory chain.

Ubiquinones have been shown to inhibit mPTP opening to a greater extent than CsA ${ }^{28}$. The proximity between the urea and phenyl motifs of C31 would be compatible with a shared mPTP target with ubiquinones. As shown in Figure 5E, ubiquinone $0\left(\mathrm{Ub}_{0}\right)$, the most efficient ubiquinone, strongly increased the calcium retention capacity of liver mitochondria isolated from wild-type mice, but had no effect in liver mitochondria isolated from Ppif ${ }^{/-}$mice (Figure 5E). This result indicates that the additional, CypD-independent effect of C31 on mPTP opening is not ubiquinone-like. 
Because it has been reported that inhibiting complex I with rotenone limits mPTP opening $^{29}$, we investigated whether $\mathrm{C} 31$ has an effect on respiratory chain functions. C31 did not alter substrate-dependent respiration rates or ADP-induced $\mathrm{O}_{2}$ consumption, suggesting no C31-induced alteration of mitochondrial respiration (Supplementary Figure 2). No drop in the electron transport chain activity (lower $\Delta \Psi \mathrm{m}$ and slower calcium absorption) was detected in our experiments. Thus, the greater potency of $\mathrm{C} 31$ as compared to CsA in inhibiting mPTP opening is not related to an interaction with mitochondrial complex I or the respiratory chain.

\section{SMCypI compound C31 inhibits mPTP opening in vivo in mice}

Anesthetized mice were infused with increasing doses of C31 for 3 min and sacrificed 2 min later. Liver mitochondria were isolated and their calcium retention capacity was measured. Mitochondria isolated from C31-treated mouse livers exhibited higher calcium retention capacities than those from vehicle-treated mice, and this effect was dose-dependent. The effect of C31 was more potent than that of CsA (Figure 6A). This result, showing in vivo inhibition of mPTP opening by SMCypI compound C31, indicates that $\mathrm{C} 31$ reaches its mitochondrial target after infusion in living mice.

SMCypI compound C31 bears mito- and hepatoprotective properties during experimental

\section{liver ischemia-reperfusion in mice}

$$
\text { Our final series of experiments aimed at demonstrating the protective effect of SMCypI }
$$
compound $\mathrm{C} 31$ in an experimental murine model of hepatic ischemia-reperfusion injury. First, mice were subjected to $60 \mathrm{~min}$ of liver ischemia followed by $10 \mathrm{~min}$ of reperfusion. Ischemiareperfusion reduced the calcium retention capacity of isolated mouse mitochondria by $59 \%$ $(96.1 \pm 4.5$ to $39.0 \pm 2.7 \mathrm{nmol} / \mathrm{mg}$ of protein; $\mathrm{p}<0.001)$. This reduction was associated with an 
alteration of oxidative phosphorylation, as demonstrated by a $31 \%$ decrease of the rate of ADPstimulated respiration $(\mathrm{p}=0.0016$ vs Sham) and a 52\% decrease of the respiratory control ratio $(\mathrm{p}=0.0026 \mathrm{vs}$ Sham) (Figure 6B). Infusion of the most effective dose of C31 (150 mg/kg) $1 \mathrm{~min}$ before and during the first 8 min of reperfusion restored normal calcium retention capacity $(84.9 \pm 8.9 \mathrm{nmol} / \mathrm{mg}$ of protein). The protective effect of $\mathrm{C} 31$ also translated into a restoration of oxidative phosphorylation parameters (Figure 6B).

Secondly, the hepatoprotective effect of C31 was assessed in mice subjected to $60 \mathrm{~min}$ of liver ischemia, followed by 60 min of reperfusion. Blood samples were collected for ALT and AST level measurement and livers were excised for liver histology assessment (Figure 7A). C31 treatment significantly reduced ischemia-reperfusion-induced ALT elevation at the end of reperfusion (mean ALT levels: 2,297 2290 versus 4,805 $\pm 1,088$ in C31-treated and vehicletreated mice, respectively; $\mathrm{p}=0.0375$ ) (Figure 7B). The effect of $\mathrm{C} 31$ on serum ALT levels was significantly greater than that of CsA, which was not significantly different from that of the vehicle (data not shown). C31 also substantially reduced AST elevation, but the difference did not reach significance (Supplementary Figure 3).

As shown in Figures 7C, 7D and 7E, C31 protected mouse livers from hepatic damages induced by ischemia-reperfusion. Indeed, $60 \mathrm{~min}$ after reperfusion, hepatic clarification areas that measure hepatocyte ballooning degeneration, a feature of acute liver injury (Figure 7D), covered a significantly smaller surface in C31-treated than in vehicle-treated mice $(30.4 \pm 4.3 \%$ versus $50.7 \pm 3.2 \%$ of liver section surface, respectively; $\mathrm{p}=0.0012$ ). CsA also significantly reduced the surface of hepatic clarification in the model, to an extent similar to the effect of C31 (data not shown).

These findings indicate that SMCypI compound C31 exerts both mito- and hepatoprotective effects in mice exposed to ischemia-reperfusion.

\section{Discussion}


limited therapeutic options. Ischemia-reperfusion injury results in great part from hepatic mitochondrial dysfunction related to abnormal mPTP opening. CypD is an important regulator 543 of the mPTP complex that plays a key role in its opening in several pathological conditions, including hepatic ischemia-reperfusion ${ }^{11}{ }^{14}$. Indeed, CypD translocation to the inner mitochondrial membrane triggers mPTP opening and promotes cell death. Both effects can be prevented by pharmacological CypD inhibitors or CypD knock-out ${ }^{27}, 30-32$. Thus, CypD represents an attractive target for inhibition of mPTP opening and hepatocellular protection in 548 the context of hepatic ischemia-reperfusion injury.

We designed a new family of SMCypIs made of two linked fragments binding the cyclophilin catalytic and gatekeeper pockets, respectively ${ }^{19}$. Our aim was to develop CypA inhibitors with antiviral activity against the hepatitis C virus. We showed that our SMCypIs bind CypA and potently inhibit both CypA PPIase activity and the replication of hepatitis C

553 virus and related viruses in cell culture ${ }^{19,33}$. Because the different cyclophilins are structurally 554 very close (essentially differing by their cellular localizations and functions), we assessed whether the new SMCypIs inhibit CypD activity. As shown here, their inhibitory activity was potent and concentration-dependent. SMCypI compound C31 was the most potent CypD inhibitor in our experiments. Its cytotoxic concentration $50 \%$ was $>100 \mu \mathrm{M}$ in Huh7 cells, 558 suggesting a favorable therapeutic index ${ }^{19}$. We also showed that $\mathrm{C} 31$ binds both the catalytic 559 and gatekeeper pockets of Cyp D.

The ability of our SMCypIs to inhibit mPTP opening and its mitochondrial

561 consequences in a concentration-dependent manner correlated with their ability to block PPIase 562 activity. Inhibition of mPTP opening was observed in different models and conditions, 563 including energized and de-energized ones. This suggests that the SMCypIs act directly on 564 CypD and mPTP opening, downstream of the site of action of the inducers used in the 
experiments. Importantly, this effect was observed in both human and mouse primary hepatocytes, a result validating the human relevance of our findings.

Although the CypD affinity of the SMCypIs was lower than that of CsA, C31 achieved more potent $\mathrm{mPTP}$ opening inhibition than $\mathrm{CsA}$ at its maximum soluble concentration in the medium. Because non-immunosuppressive derivatives of CsA, e.g., PKF220-384 or NIM811, have been shown to be equipotent to CsA in inhibiting mPTP opening ${ }^{32}$, these compounds are also likely to have a weaker effect on mPTP opening than C31 (not tested). Importantly, our findings suggest that the additional effect of C31 on mPTP opening as compared to CsA is independent from CypD inhibition. Indeed, first, the concentrations of CsA and C31 that fully inhibited CypD PPIase activity retained the same amount of calcium in mitochondria; secondly, CsA did not alter the maximal calcium retention capacity induced by $\mathrm{C} 31$; thirdly, $\mathrm{C} 31$ had an effect on calcium accumulation in liver mitochondria isolated from Ppif ${ }^{/-}$mice that do not express CypD.

The fact that CsA fully inhibited CypD PPIase activity in isolated mouse mitochondria (Supplementary Figure 3) suggests that the additional effect of C31 as compared to CsA is not related to its ability to inhibit PPIase activity. Because the structure and functioning of mPTP remain largely unknown, we could not identify the second target of C31 responsible for its greater potency as compared to CsA. We could however rule out an alteration of mitochondrial respiration $^{29}$ or a ubiquinone-like mechanism ${ }^{28}$. Many potential components or regulators of the $\mathrm{mPTP}$, such as the recently identified $\mathrm{SPG}^{34}$, could be involved, while many questions remain unanswered: is mPTP organized as a true physical channel? Is opening just a different state of the mitochondrial membrane with increased permeability? Which cellular components are actual constituents of the mPTP? Which ones only interact with it and/or regulate it? What are the mechanisms involved? Answering these questions was beyond the scope of our study, but we are confident that the new family of SMCypIs will be helpful in future mechanistic studies aimed at exploring these questions. Altogether, our result identify SMCypI C31 as a 
591

592

particularly promising mPTP opening blocking agent, because its effect on mPTP opening related to CypD PPIase activity inhibition is enhanced by another complementary mechanism. Key results in this study were obtained in vivo in murine models. We showed that C31 rapidly reaches its target after systemic administration. This is particularly important in the context of ischemia-reperfusion, because mPTP was reported to open within the first minutes of reperfusion after ischemia ${ }^{8,35}$. For this reason, proof-of-concept efficacy experiments were performed in vivo in a murine model of ischemia-reperfusion. The administration of C31 at reperfusion restored the mitochondrial function. In addition, ALT elevations and hepatic damage were significantly reduced in C31-treated mice, proving the hepatoprotective properties of the new SMCypI, as a result of mPTP opening inhibition.

In this study, liver damage was assessed by measuring "hepatocyte clarification", i.e. hepatocyte ballooning degeneration, a feature of severe liver cell injury involving ATP depletion and a rise in intracellular calcium concentrations leading to a loss of cell volume control by plasma membranes and a disruption of the hepatocyte intermediate filament network. Hepatocyte ballooning degeneration has been observed in the early post-transplant period ${ }^{36-3839}$, and described as an important morphological characteristic of ischemia-reperfusion injury measuring the degree of liver damage ${ }^{40-42}$. As shown in Figure 7D in our model, hepatocyte ballooning degeneration was characterized by hepatocellular swelling, cytoplasm vacuolization and blebbing of the cell membrane. It was significantly improved by C31 administration in our murine model of ischemia-reperfusion injury. Because the half-life of C31 is short, requiring continuous infusion, it was not possible to determine how long the protective effects might last. Chemical, galenic and pharmacological optimization of the compounds is in progress and studies with more stable derivatives will be conducted to address this important question as part of future preclinical development.

In summary, we showed that our new family of small-molecule, non-peptidic cyclophilin inhibitors binds CypD and inhibits its PPIase activity, and that this effect is, at least 
617

618

619

620

621

622

623

624

625

626

627

628

629

630

631

632

633

in part, responsible for their concentration-dependent inhibitory effect on calcium-induced swelling due to mPTP opening in both human and mouse hepatocytes. We also showed that the most potent SMCypI, compound C31, exerts an additional effect on mPTP opening, independent of its inhibitory effect on CypD, making it a promising pharmacological agent for hepatocellular protection in the context of diseases involving mitochondrial dysfunction related to abnormal mPTP opening. Finally, our study is the first to provide the in vivo proof-of-concept of mitochondrial and hepatocellular protection by SMCypI compound C31 in an experimental model of ischemia-reperfusion injury.

The new family of SMCypIs appears as a promising candidate for new drug development in the indication of hepatic protection in the context of warm ischemia-reperfusion after liver surgery. Whether similar protection can be obtained in the context of cold ischemiareperfusion related to orthotopic liver transplantation remains to be assessed. Other applications in liver and non-hepatic diseases related to mPTP opening involving CypD deserve to be explored. They include chronic alcohol consumption, which enhances sensitivity to calciummediated mPTP opening and increases CypD expression ${ }^{43}$, drug-induced liver injury, myocardial ischemia-reperfusion, brain injury and neurodegenerative disorders. 
634

635

636

637

638

639

640

641

642

643

644

645

646

647

648

649

650

651

652

653

654

655

656

657

658

\section{References}

1. Konishi T, Lentsch AB. Hepatic ischemia/reperfusion: mechanisms of tissue injury, repair, and regeneration. Gene Expr 2017;17:277-287.

2. Cannistra M, Ruggiero M, Zullo A, et al. Hepatic ischemia reperfusion injury: a systematic review of literature and the role of current drugs and biomarkers. Int J Surg 2016;33 Suppl 1:S57-70.

3. Go KL, Lee S, Zendejas I, et al. Mitochondrial dysfunction and autophagy in hepatic ischemia/reperfusion injury. Biomed Res Int 2015;2015:183469.

4. Halestrap AP. What is the mitochondrial permeability transition pore? J Mol Cell Cardiol 2009;46:821-31.

5. Friberg H, Wieloch T. Mitochondrial permeability transition in acute neurodegeneration. Biochimie 2002;84:241-50.

6. Kim JS, He L, Qian T, et al. Role of the mitochondrial permeability transition in apoptotic and necrotic death after ischemia/reperfusion injury to hepatocytes. Curr Mol Med 2003;3:527-35.

7. Rauen U, de Groot H. New insights into the cellular and molecular mechanisms of cold storage injury. J Investig Med 2004;52:299-309.

8. Halestrap AP. A pore way to die: the role of mitochondria in reperfusion injury and cardioprotection. Biochem Soc Trans 2010;38:841-60.

9. Rao VK, Carlson EA, Yan SS. Mitochondrial permeability transition pore is a potential drug target for neurodegeneration. Biochim Biophys Acta 2014;1842:1267-72.

10. Jaeschke H, McGill MR, Ramachandran A. Oxidant stress, mitochondria, and cell death mechanisms in drug-induced liver injury: lessons learned from acetaminophen hepatotoxicity. Drug Metab Rev 2012;44:88-106. 
659

660

661

662

663

664

665

666

667

668

669

670

671

672

673

674

675

676

677

678

679

680

681

682

11. Giorgio V, von Stockum S, Antoniel M, et al. Dimers of mitochondrial ATP synthase form the permeability transition pore. Proc Natl Acad Sci USA 2013;110:5887-92.

12. Wang P, Heitman J. The cyclophilins. Genome Biol 2005;6:226.

13. Davis TL, Walker JR, Campagna-Slater V, et al. Structural and biochemical characterization of the human cyclophilin family of peptidyl-prolyl isomerases. PLoS Biol 2010;8:e1000439.

14. Javadov S, Kuznetsov A. Mitochondrial permeability transition and cell death: the role of cyclophilin D. Front Physiol 2013;4:76.

15. Griffiths EJ, Halestrap AP. Protection by cyclosporin A of ischemia/reperfusion-induced damage in isolated rat hearts. J Mol Cell Cardiol 1993;25:1461-9.

16. Matsuda S, Koyasu S. Mechanisms of action of cyclosporine. Immunopharmacology 2000;47:119-25.

17. Azzolin L, Antolini N, Calderan A, et al. Antamanide, a derivative of Amanita phalloides, is a novel inhibitor of the mitochondrial permeability transition pore. PLoS One 2011;6:e16280.

18. Piot C, Croisille P, Staat P, et al. Effect of cyclosporine on reperfusion injury in acute myocardial infarction. N Engl J Med 2008;359:473-81.

19. Ahmed-Belkacem A, Colliandre L, Ahnou N, et al. Fragment-based discovery of a new family of non-peptidic small-molecule cyclophilin inhibitors with potent antiviral activities. Nat Commun 2016;7:12777.

20. Pons JL, Labesse G. @TOME-2: a new pipeline for comparative modeling of proteinligand complexes. Nucleic Acids Res 2009;37:W485-91.

21. Miteva MA, Guyon F, Tuffery P. Frog2: efficient 3D conformation ensemble generator for small compounds. Nucleic Acids Res 2010;38:W622-7. 
683

684

685

686

687

688

689

690

691

692

693

694

695

696

697

698

699

700

701

702

703

704

705

706

707

708

22. Fontaine E, Eriksson O, Ichas F, et al. Regulation of the permeability transition pore in skeletal muscle mitochondria. Modulation by electron flow through the respiratory chain complex I. J Biol Chem 1998;273:12662-8.

23. Seglen PO. Preparation of isolated rat liver cells. Methods Cell Biol 1976;13:29-83.

24. Petronilli V, Miotto G, Canton M, et al. Transient and long-lasting openings of the mitochondrial permeability transition pore can be monitored directly in intact cells by changes in mitochondrial calcein fluorescence. Biophys J 1999;76:725-34.

25. Petronilli V, Penzo D, Scorrano L, et al. The mitochondrial permeability transition, release of cytochrome $\mathrm{C}$ and cell death. Correlation with the duration of pore openings in situ. J Biol Chem 2001;276:12030-4.

26. Bankhead P, Loughrey MB, Fernandez JA, et al. QuPath: open source software for digital pathology image analysis. Sci Rep 2017;7:16878.

27. Baines CP, Kaiser RA, Purcell NH, et al. Loss of cyclophilin D reveals a critical role for mitochondrial permeability transition in cell death. Nature 2005;434:658-62.

28. Walter L, Miyoshi H, Leverve X, et al. Regulation of the mitochondrial permeability transition pore by ubiquinone analogs. A progress report. Free Radic Res 2002;36:40512.

29. Li B, Chauvin C, De Paulis D, et al. Inhibition of complex I regulates the mitochondrial permeability transition through a phosphate-sensitive inhibitory site masked by cyclophilin D. Biochim Biophys Acta 2012;1817:1628-34.

30. Nakagawa T, Shimizu S, Watanabe T, et al. Cyclophilin D-dependent mitochondrial permeability transition regulates some necrotic but not apoptotic cell death. Nature $2005 ; 434: 652-8$.

31. Hansson MJ, Mattiasson G, Mansson R, et al. The nonimmunosuppressive cyclosporin analogs NIM811 and UNIL025 display nanomolar potencies on permeability transition in brain-derived mitochondria. J Bioenerg Biomembr 2004;36:407-13. 
32. Waldmeier PC, Feldtrauer JJ, Qian T, et al. Inhibition of the mitochondrial permeability transition by the nonimmunosuppressive cyclosporin derivative NIM811. Mol Pharmacol 2002;62:22-9.

33. Nevers Q, Ruiz I, Ahnou N, et al. Characterization of the anti-hepatitis C virus activity of new nonpeptidic small-molecule cyclophilin inhibitors with the potential for broad antiFlaviviridae activity. Antimicrob Agents Chemother 2018;62:e00126-18.

34. Shanmughapriya S, Rajan S, Hoffman NE, et al. SPG7 is an essential and conserved component of the mitochondrial permeability transition pore. Mol Cell 2015;60:47-62.

35. Glantzounis GK, Salacinski HJ, Yang W, et al. The contemporary role of antioxidant therapy in attenuating liver ischemia-reperfusion injury: a review. Liver Transpl 2005;11:1031-47.

36. Lefkowitch JH. Scheuer's Liver biopsy interpretation. Edinburgh: Elsevier, 2016.

37. Neil DA, Hubscher SG. Delay in diagnosis: a factor in the poor outcome of late acute rejection of liver allografts. Transplant Proc 2001;33:1525-6.

38. Hubscher SG. Histological findings in liver allograft rejection: new insights into the pathogenesis of hepatocellular damage in liver allografts. Histopathology 1991;18:37783.

39. Khettry U, Backer A, Ayata G, et al. Centrilobular histopathologic changes in liver transplant biopsies. Hum Pathol 2002;33:270-6.

40. Datta G, Fuller BJ, Davidson BR. Molecular mechanisms of liver ischemia reperfusion injury: insights from transgenic knockout models. World J Gastroenterol 2013;19:168398.

41. Saeed WK, Jun DW, Jang K, et al. Does necroptosis have a crucial role in hepatic ischemia-reperfusion injury? PLoS One 2017;12:e0184752.

42. Arab HA, Sasani F, Rafiee MH, et al. Histological and biochemical alterations in earlystage lobar ischemia-reperfusion in rat liver. World J Gastroenterol 2009;15:1951-7. 
735 43. King AL, Swain TM, Dickinson DA, et al. Chronic ethanol consumption enhances sensitivity to $\mathrm{Ca}(2+)$-mediated opening of the mitochondrial permeability transition pore and increases cyclophilin D in liver. Am J Physiol Gastrointest Liver Physiol 2010;299:G954-66.

739

740 


\section{Figure legends}

Figure 1. (A) Molecular modeling and docking of SMCypI compound C31 bound to the catalytic site (left) and gatekeeper pocket (right) of CypD. (B) Concentration-dependent inhibitory effect of SMCypI C31 on isolated mouse liver mitochondrial swelling. Swelling was induced by $50 \mu \mathrm{M}$ calcium in the absence of inhibitors, in the presence of $1 \mu \mathrm{M}$ CsA (positive control of inhibition) or in the presence of increasing $(0.1$ to $10 \mu \mathrm{M})$ concentrations of $\mathrm{C} 31$. No calcium was added in the negative control. The $\mathrm{x}$ axis indicates times of measurement; the $\mathrm{y}$ axis shows dynamic changes in absorbance at $540 \mathrm{~nm}$ (A540) reflecting changes in mitochondrial swelling. (C) Relationship between SMCypI inhibition of CypD PPIase activity in an enzyme assay and swelling of isolated mouse liver mitochondria.

Figure 2. Inhibitory effect of SMCypI C31 on mitochondrial swelling triggered by mPTP opening inducers in isolated mouse mitochondria incubated in the presence of $\mathrm{Ca}^{2+}$. The $\mathrm{x}$ axis indicates times of measurement; the y axis shows dynamic changes in absorbance at $540 \mathrm{~nm}$ (A $\mathrm{A}_{540}$ ) reflecting changes in mitochondrial swelling. (A) mPTP opening induced by $40 \mu \mathrm{M}$ tertbutyl hydroperoxide $(t-\mathrm{BH})$ in energized mitochondria. (B) mPTP opening induced by $100 \mu \mathrm{M}$ carboxyatractyloside (CAT) in energized mitochondria. (C) mPTP opening induced by $1 \mu \mathrm{M}$ A23187 (A23) in non-energized mitochondria. (D) mPTP opening induced by $10 \mu \mathrm{M}$ phenylarsine oxide (PAO) in non-energized mitochondria.

Figure 3. Inhibition of mPTP opening by CsA and SMCypI compound C31 in primary mouse and human hepatocytes. (A) Experimental procedure: hepatocytes were loaded with calcein and $\mathrm{CoCl}_{2}$ and mPTP opening was induced by the addition of $1 \mu \mathrm{M}$ of the calcium ionophore A23187 (A23), in the absence (control) or in the presence of CsA or of increasing concentrations of C31. Images were collected at 1-min intervals. Fluorescence was normalized 
to $100 \%$ of the maximal value. The results of 4 to 7 experiments were averaged. (B) Inhibition

of mPTP opening by CsA and SMCypI compound C31 in primary mouse hepatocytes. Left curves: kinetics of calcein fluorescence over time; right bar graph: calcein fluorescence measured at $30 \min \left(\# p<0.05\right.$ vs A23 alone; ${ }^{*} p<0.05$ vs control; $\dagger p<0.05$ vs CsA) (C) Inhibition of mPTP opening by CsA and SMCypI compound C31 in primary human hepatocytes. Left curves: kinetics of calcein fluorescence over time; right bar graph: calcein fluorescence measured at $30 \min (\# p<0.05$ vs A23 alone; * $p<0.05$ vs control (Ctrl); $\uparrow p<0.05$ vs CsA).

Figure 4. Calcium retention capacity of isolated mouse liver mitochondria in the presence of SMCypI compound C31 and of CsA. (A) Representative experiment showing mitochondrial calcium retention capacity in the presence of $1 \mu \mathrm{M}$ CsA or increasing concentrations of C31. Each fluorescence peak corresponds to the addition of $20 \mu \mathrm{M}$ calcium. (B) Average calcium concentrations required for mPTP opening, expressed as a percentage of the control value ( $100 \%$ represents $109 \pm 15 \mu \mathrm{M}$, as indicated by the dashed line). ${ }^{*} \mathrm{p}<0.05$ vs value observed with $0.1 \mu \mathrm{M}$ C31; $\uparrow \mathrm{p}<0.05$ vs $1 \mu \mathrm{M}$ CsA. CsA: cyclosporine A; AU: arbitrary unit; CRC: calcium retention capacity; Ctrl: control.

Figure 5. Investigation of the mechanisms underlying the more potent effect of SMCypI compound $\mathrm{C} 31$ on mitochondrial calcium retention capacity, as compared to CsA. (A) Mean \pm SEM calcium retention capacity of isolated liver mitochondria from Ppif ${ }^{/-}(\mathrm{CypD}$ knocked-out) and wild-type mice. CRC: calcium retention capacity; WT: wild-type; \#p <0.05 $v s$ wild-type control; * $\mathrm{p}<0.05$ vs $\mathrm{Ppif}^{/-}$and wild-type controls, respectively; $\uparrow \mathrm{p}<0.05$ vs CsA. (B) Representative experiment showing the calcium concentrations required for mPTP opening in liver mitochondria isolated from $\mathrm{Ppif}^{/-}$mice in the absence of compounds (control) or in the presence of $1 \mu \mathrm{M}$ CsA or $100 \mu \mathrm{M} \mathrm{C} 31$. Each fluorescence peak corresponds to the addition of 
$20 \mu \mathrm{M}$ calcium. AU: arbitrary units. (C) Concentration-dependent C31 inhibition of mitochondrial swelling induced by $500 \mu \mathrm{M}$ calcium in liver mitochondria isolated from Ppif - $^{-}$ mice. A540: absorbance at $540 \mathrm{~nm}$. (D) Effect of $100 \mu \mathrm{M} \mathrm{C} 31$ and C34 (a C31 derivative lacking the aromicity of its phenyl moiety) on mitochondrial calcium retention capacity in isolated liver mitochondria from Ppif $^{/-}$mice. Each fluorescence peak corresponds to the addition of $20 \mu \mathrm{M}$ calcium. AU: arbitrary units. (E) Left: representative experiments showing the effect of $50 \mu \mathrm{M}$ $\mathrm{C} 31$ and $50 \mu \mathrm{M}$ ubiquinone 0 on $\mathrm{mPTP}$ opening in liver mitochondria isolated from wild-type (top) and Ppif $^{/-}$(bottom) mice. Each fluorescence peak corresponds to the addition of $20 \mu \mathrm{M}$ calcium. Right: Mean \pm SEM calcium retention capacity (CRC) in the corresponding experiments. WT: wild-type; $\mathrm{Ub}_{0}$ : ubiquinone 0 ; AU: arbitrary units; ${ }^{*} \mathrm{p}<0.05$ vs control (Ctrl).

Figure 6. In vivo effect of $\mathrm{C} 31$ on $\mathrm{mPTP}$ opening and mitochondrial alterations related to liver ischemia-reperfusion. (A) Anesthetized mice were infused with vehicle (VEH), CsA or different doses of $\mathrm{C} 31$ for $3 \mathrm{~min}$ and were sacrificed 2 min later. Liver mitochondria were isolated and the calcium retention capacities (CRC) of these mitochondria are shown. $* p<0.05$ vs VEH; \#p<0.05 vs CsA. (B) The mice were subjected to 60 min of a $70 \%$ partial liver ischemia, followed by $10 \mathrm{~min}$ of reperfusion, and received either $150 \mathrm{mg} / \mathrm{kg} \mathrm{C} 31$ or vehicle (VEH). At the end of the reperfusion period, mouse livers were excised and mitochondria were isolated to assess the $\mathrm{CRC}$ (left) and mitochondrial respiration parameters (right), including the ADP-stimulated respiration rate (state 3), the substrate-dependent respiration rate (state 4) and the respiratory control ratio (RCR: state $3 /$ state 4$) .{ }^{*} p<0.05$ vs sham.

Figure 7. In vivo hepatoprotective effect of C31 in the context of liver ischemia-reperfusion. (A) The mice were subjected to $60 \mathrm{~min}$ of a $70 \%$ partial liver ischemia, followed by $60 \mathrm{~min}$ of reperfusion; they received either $150 \mathrm{mg} / \mathrm{kg} \mathrm{C} 31$ or vehicle (VEH). At the end of the reperfusion period, blood samples and mouse livers were collected for assessment of liver damage. (B) 
819 Mean \pm SEM ALT levels 60 min after reperfusion in VEH- and C31-treated animals; *p <0.05.

820 (C) Proportion of liver section surface occupied by hepatocyte clarification in VEH- and C31-

821 treated animals; ${ }^{*} \mathrm{p}<0.05$. (D) Morphological alterations of hepatocyte clarification or hepatic

822 ballooning degeneration : A shows hepatocyte swelling and cytoplasmic clarification; B shows

823 cytoplasmic vacuolisation; $\mathrm{C}$ shows diffuse cell borders, an indirect feature of blebbing cell

824 membrane. (E) Representative hematoxylin-eosin-stained liver sections from VEH- and C31-

825 treated groups.

826

827 
Fig. 1

A

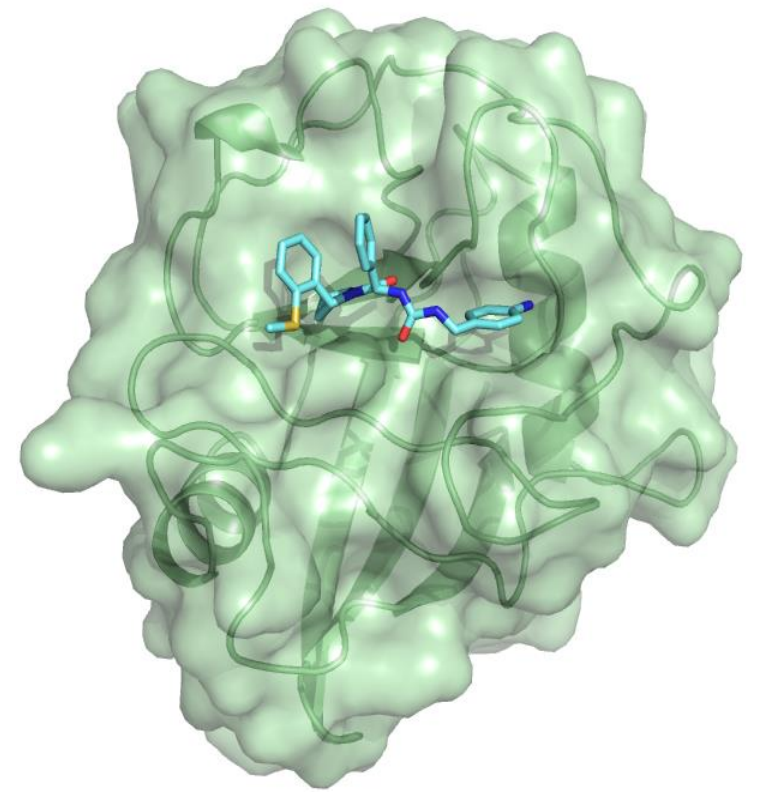

B

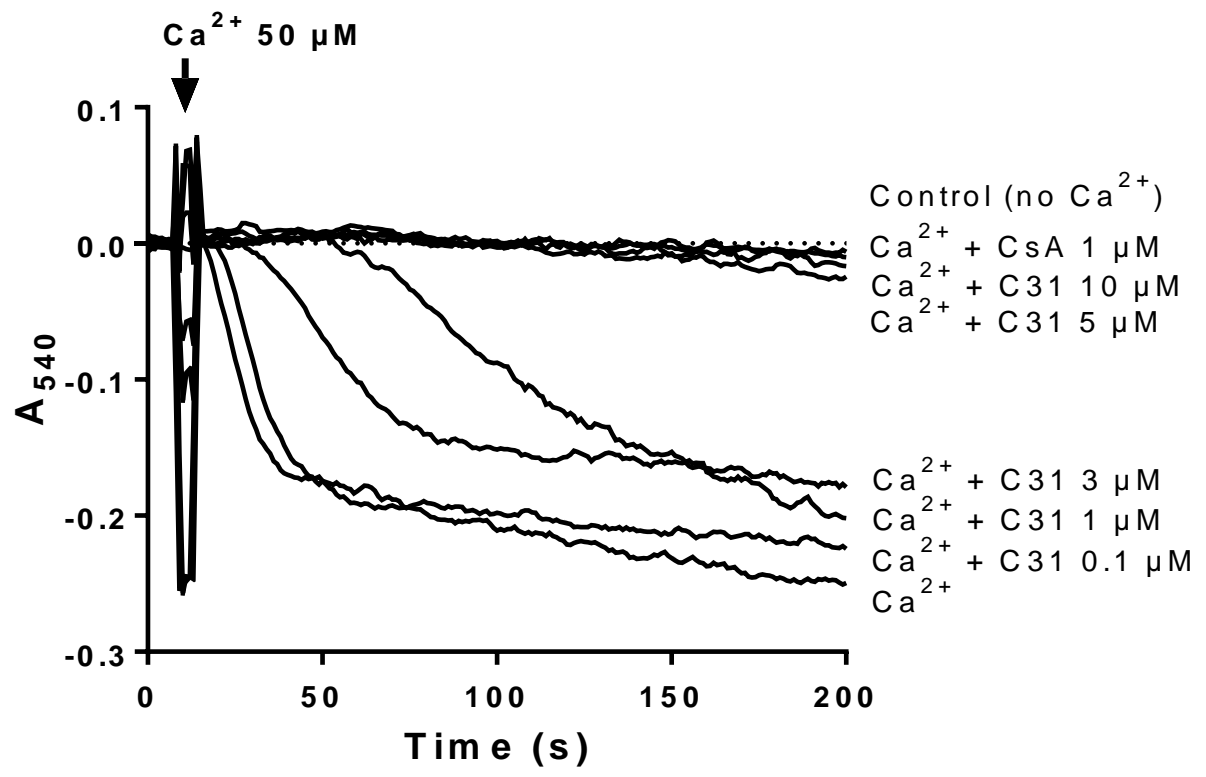

C

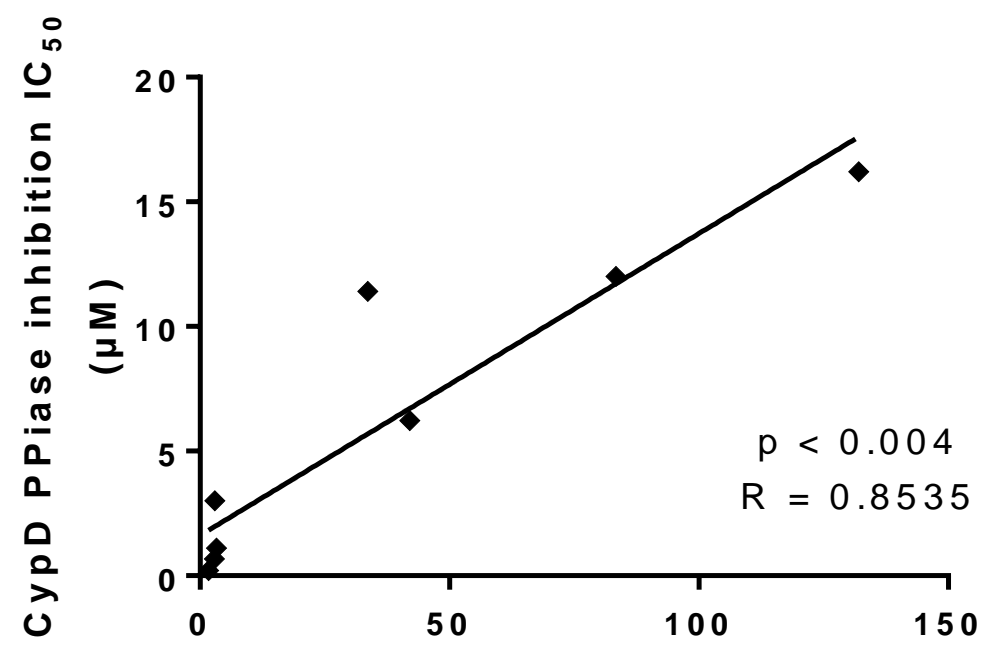

Mitochondrial swelling inhibition $I_{50}(\mu M)$ 
Fig. 2

A

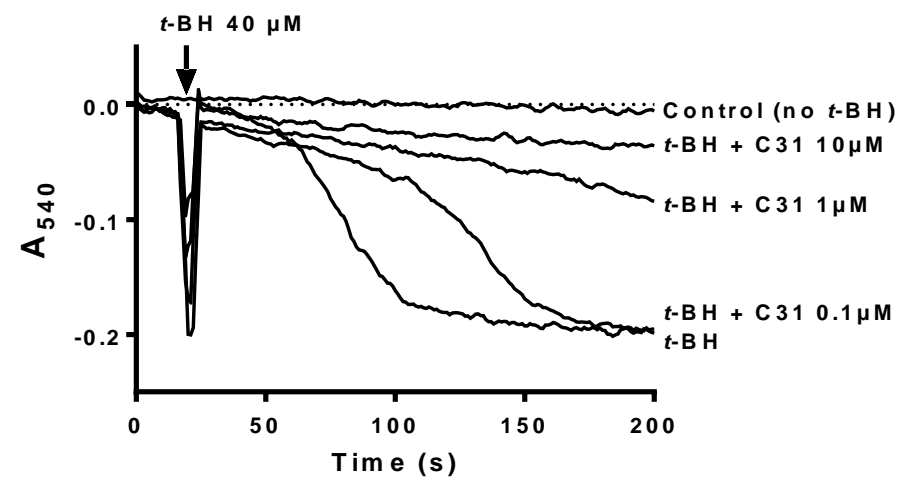

C

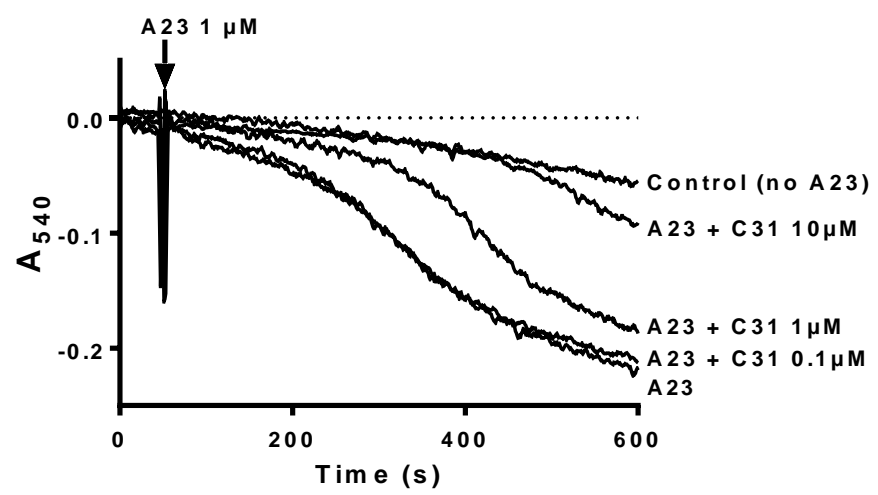

B

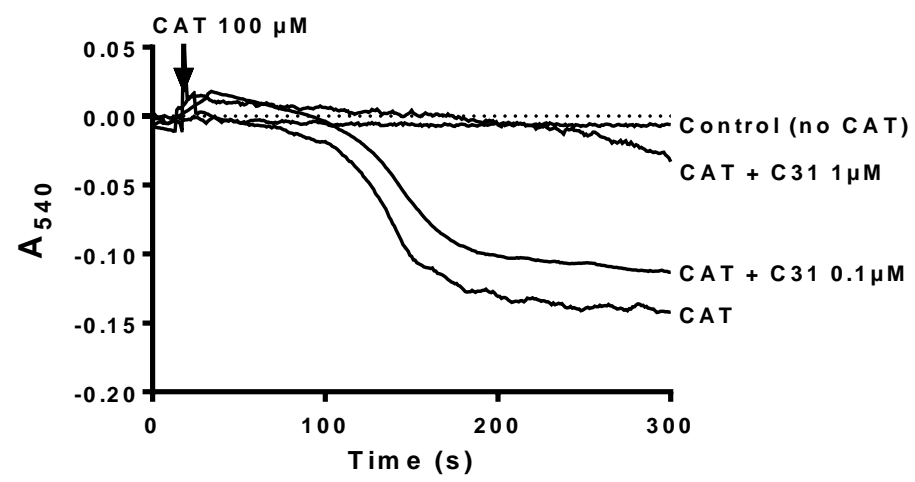

D

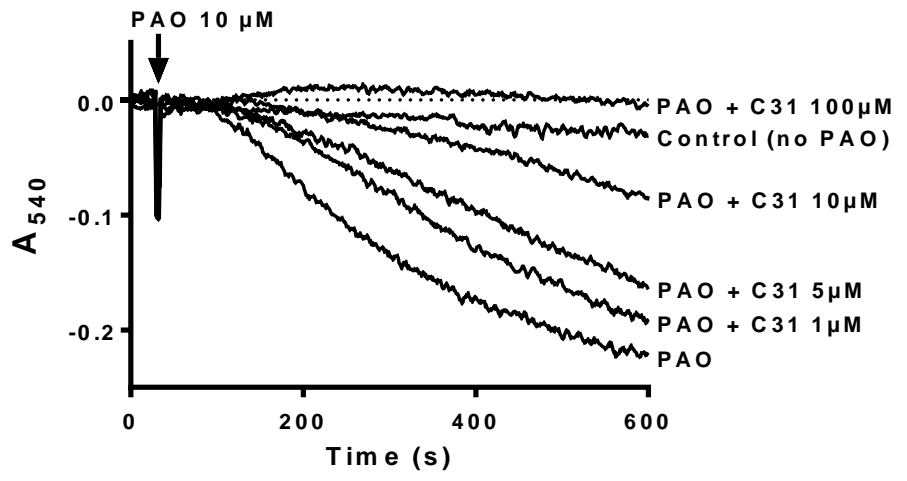


Fig. 3

A

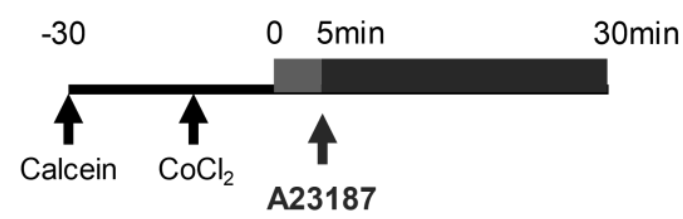

B

Primary mouse hepatocytes
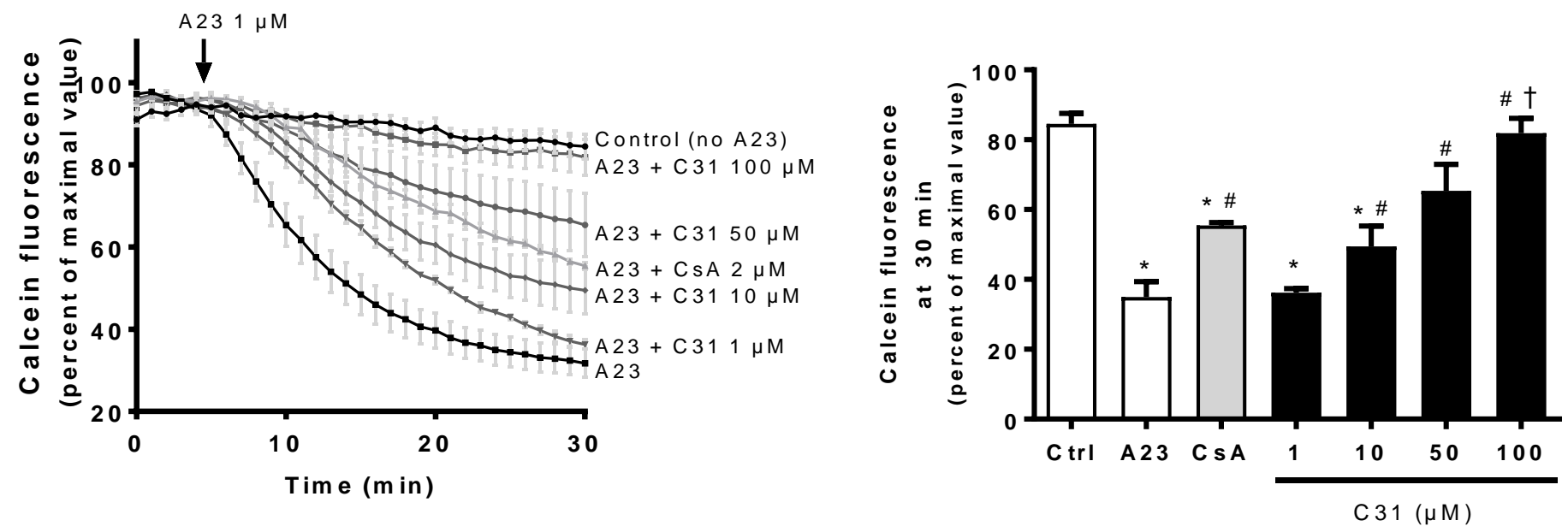

C

Primary human hepatocytes
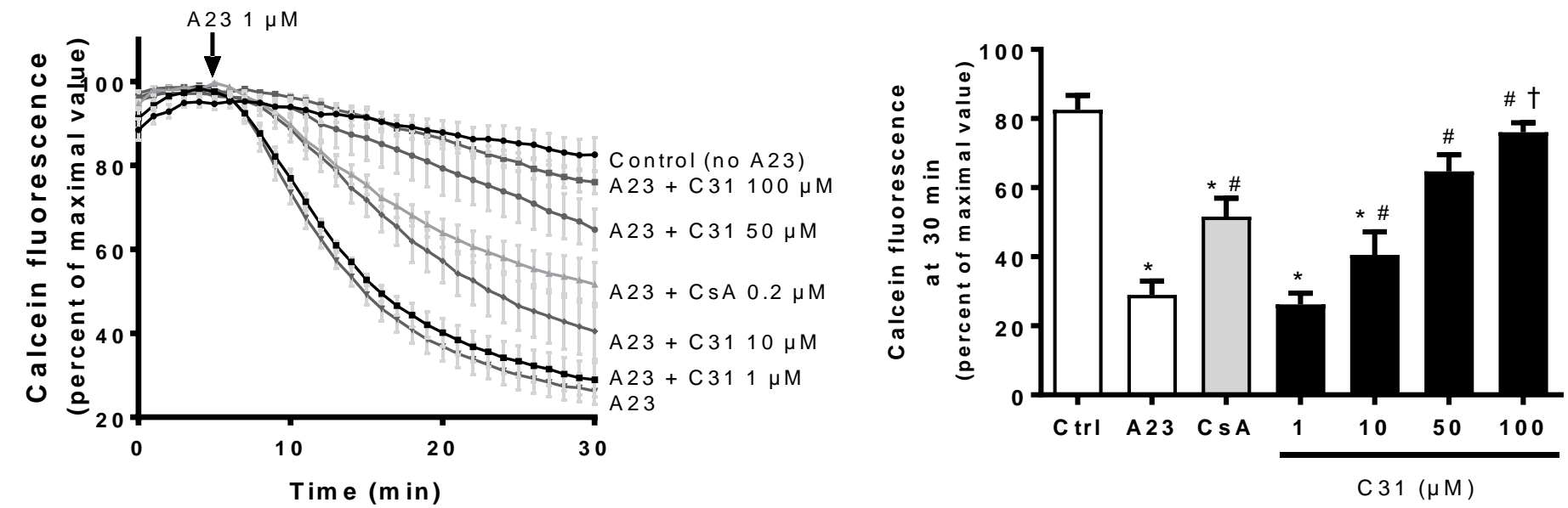
Fig. 4

A

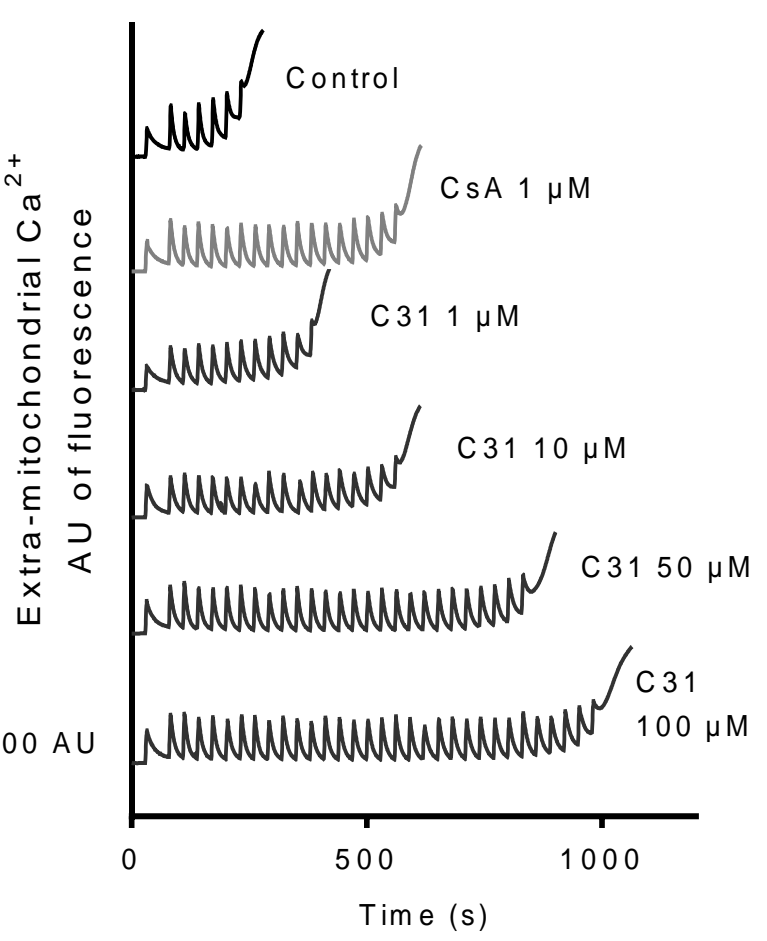

B

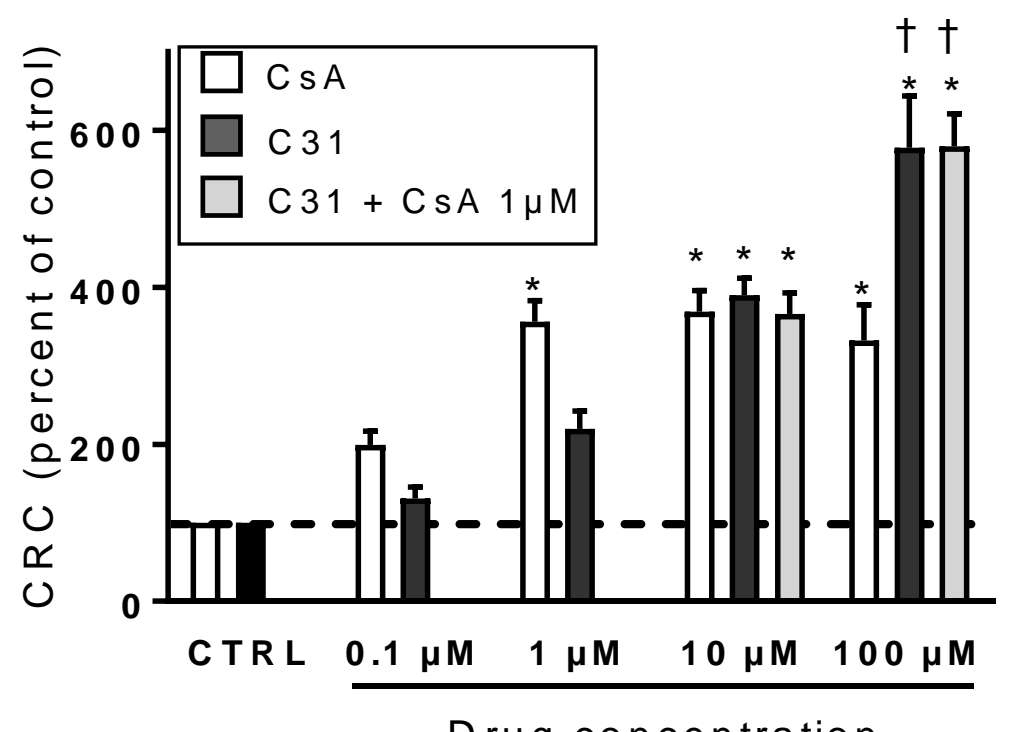

Drug concentration 
Fig. 5

A

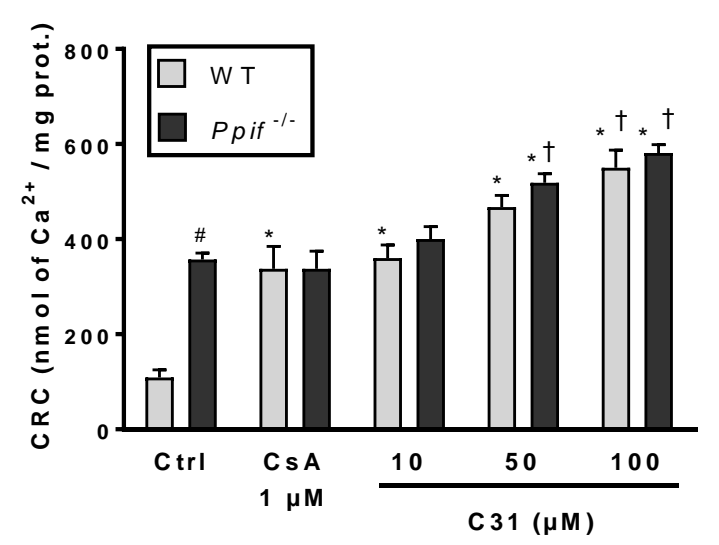

C

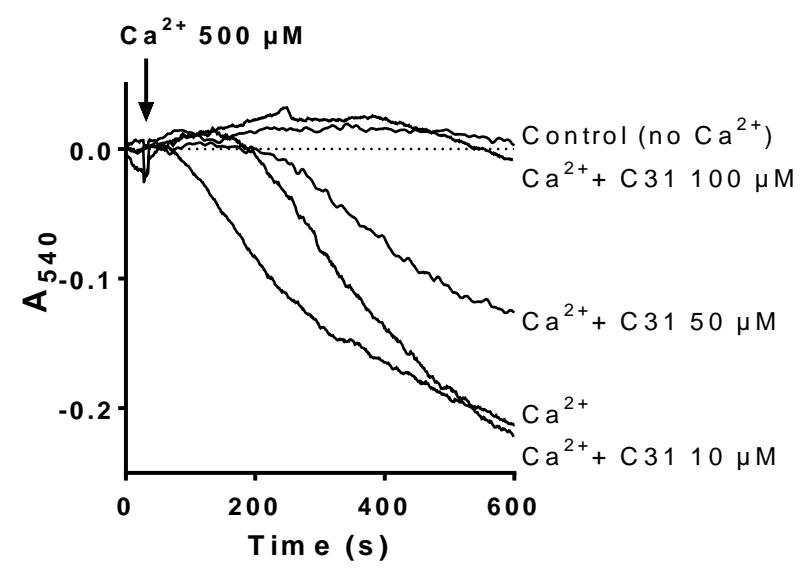

E
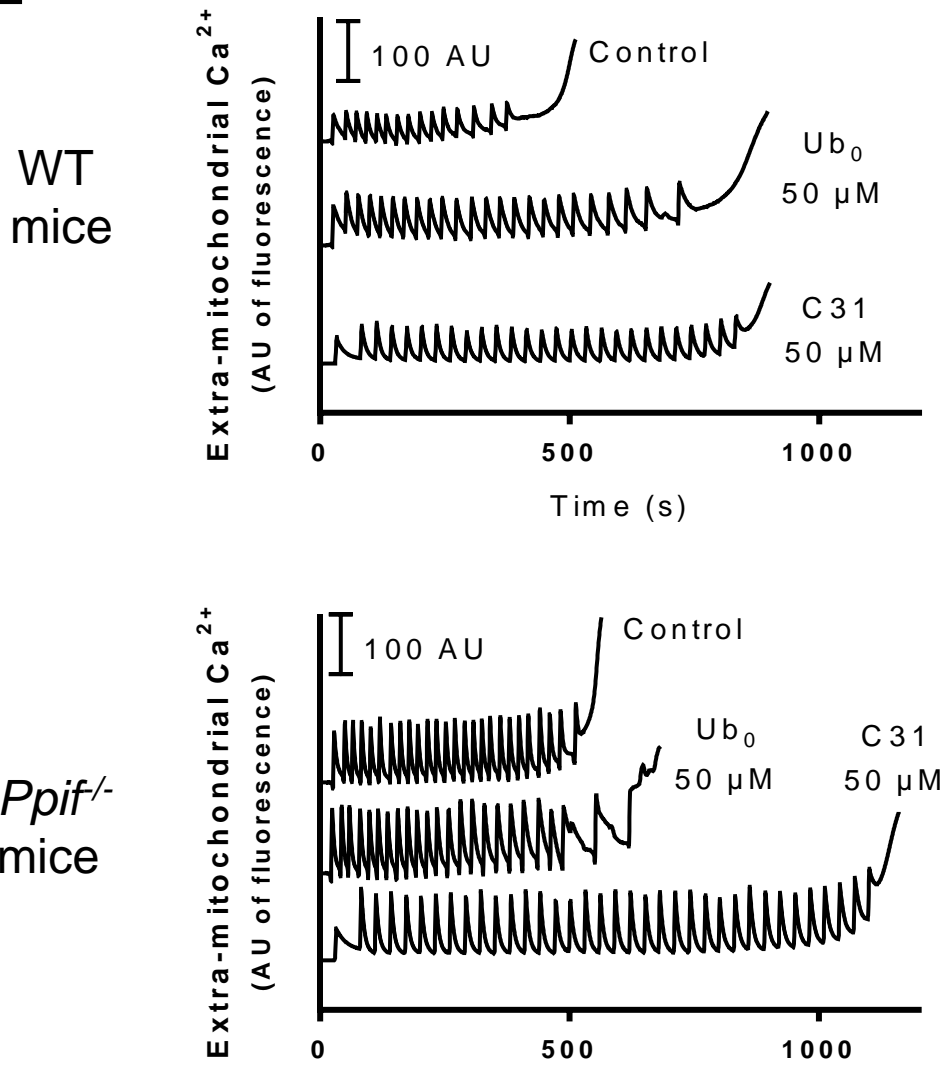

Time (s)
B

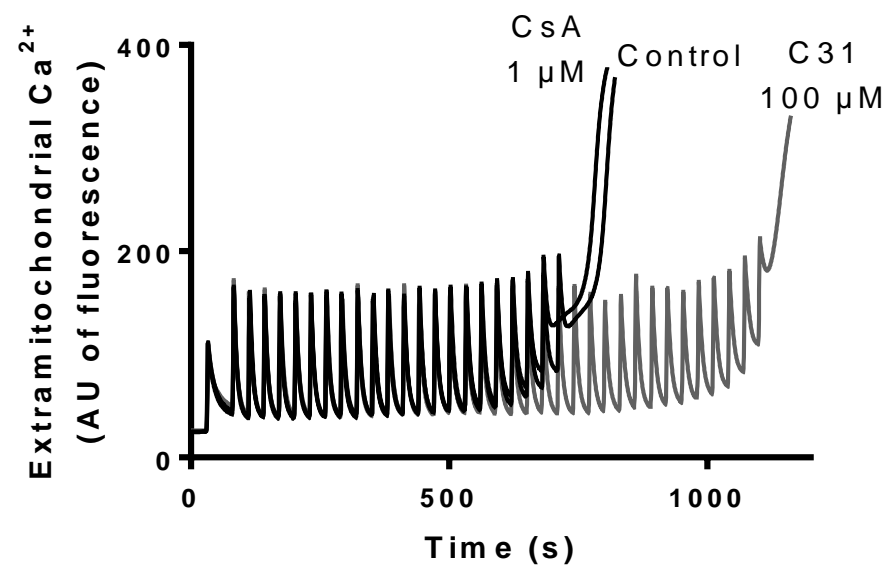

D
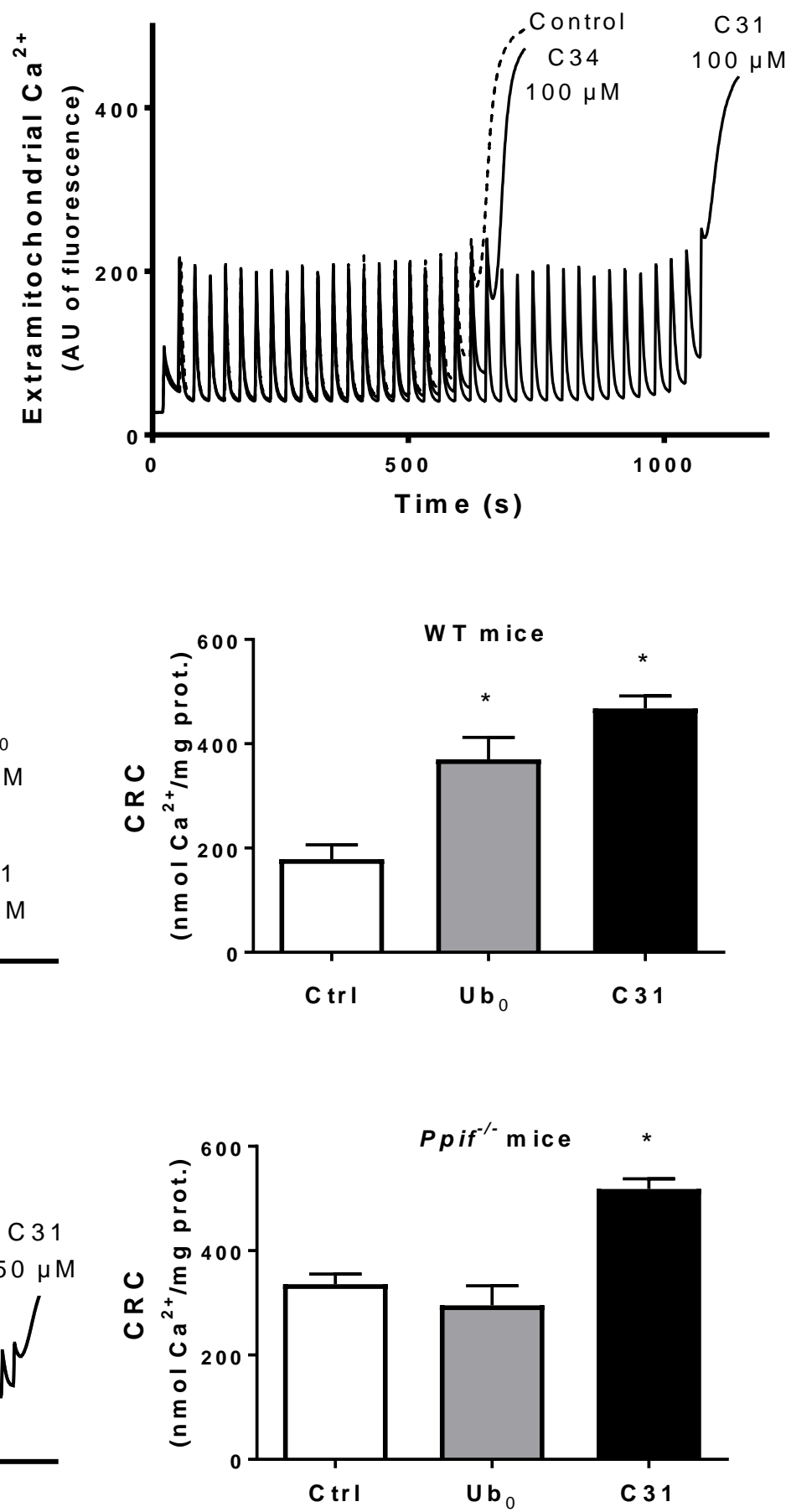
Fig. 6

A
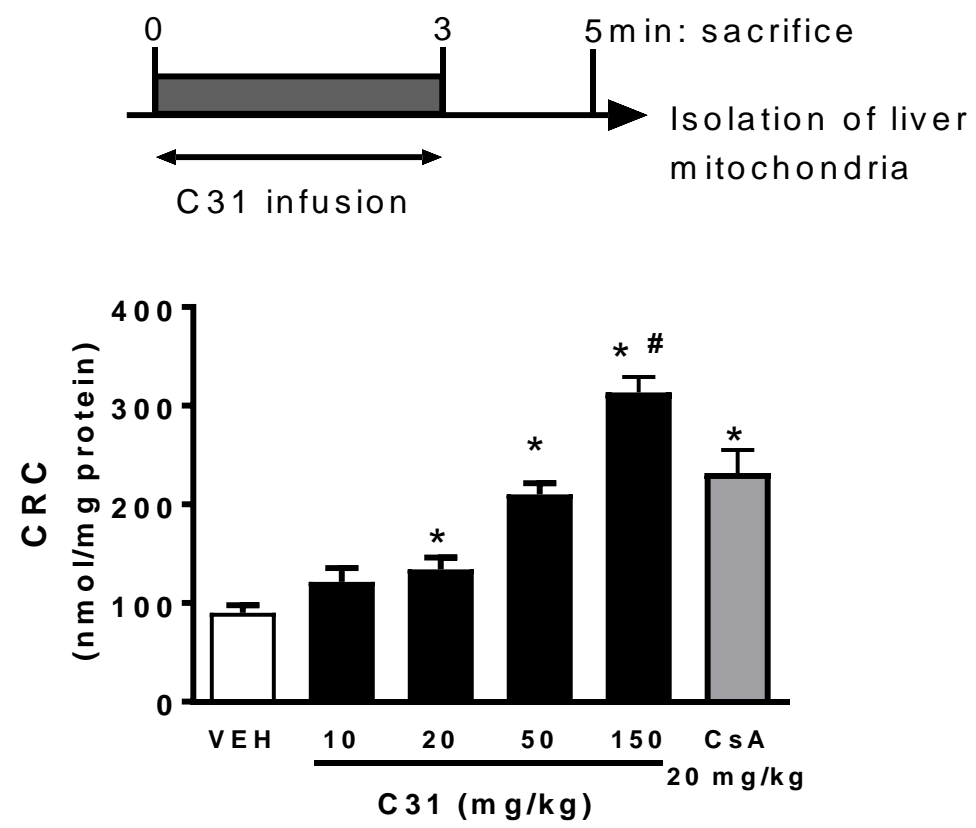

B

Reperfusion

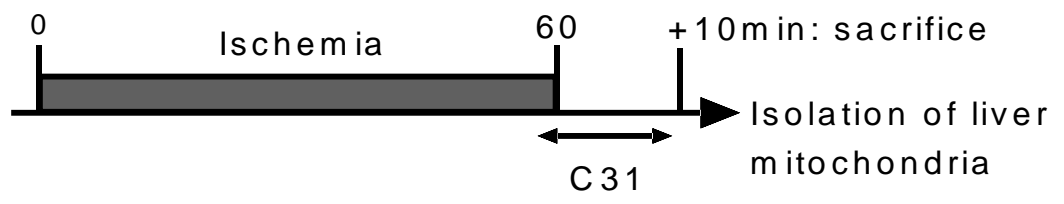

or vehicle
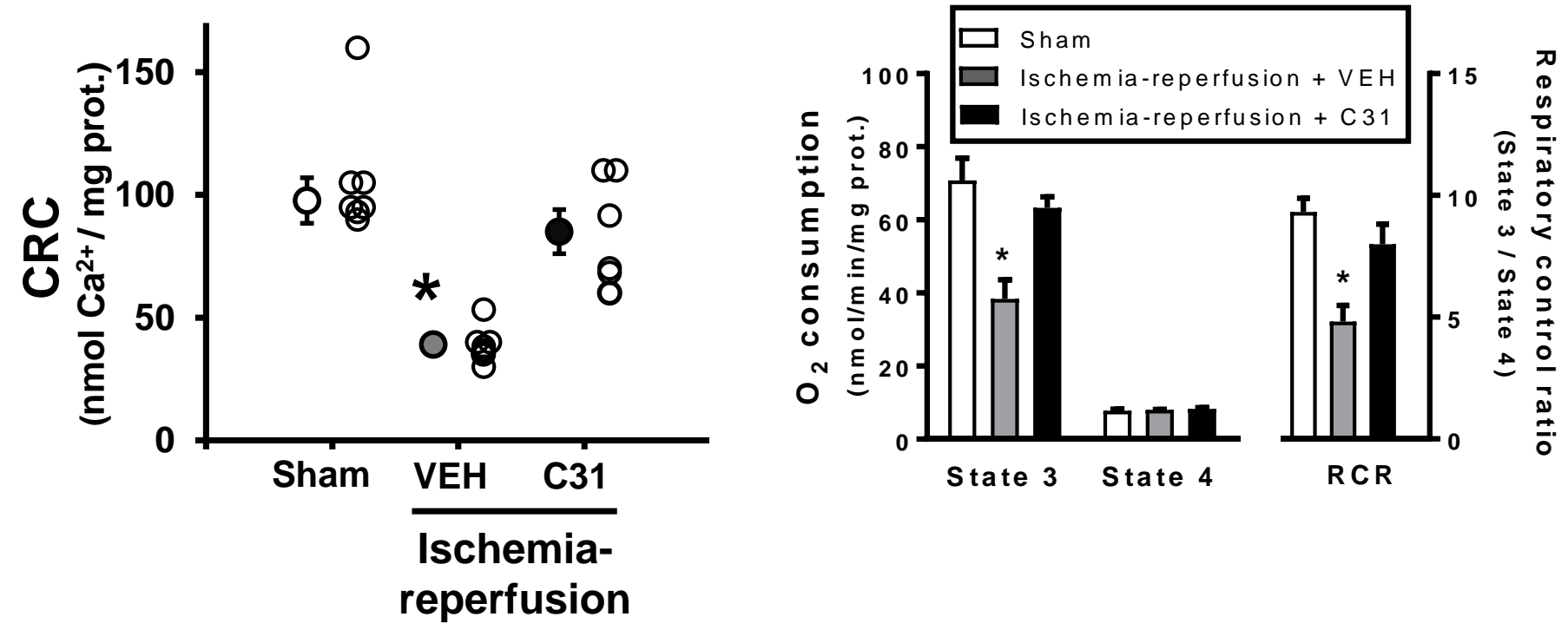

reperfusion 
Fig. 7

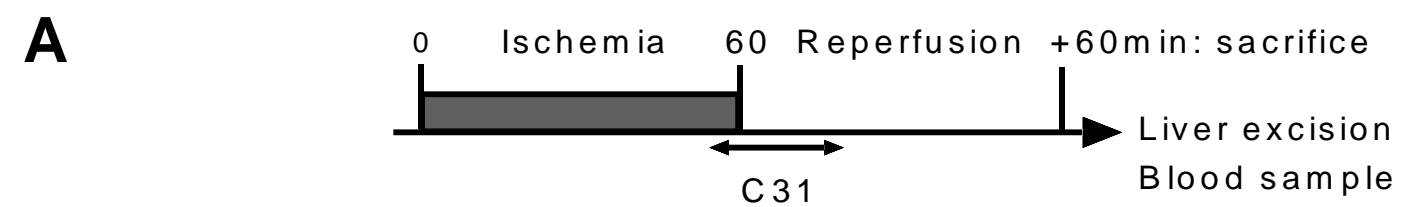

or vehicle

B

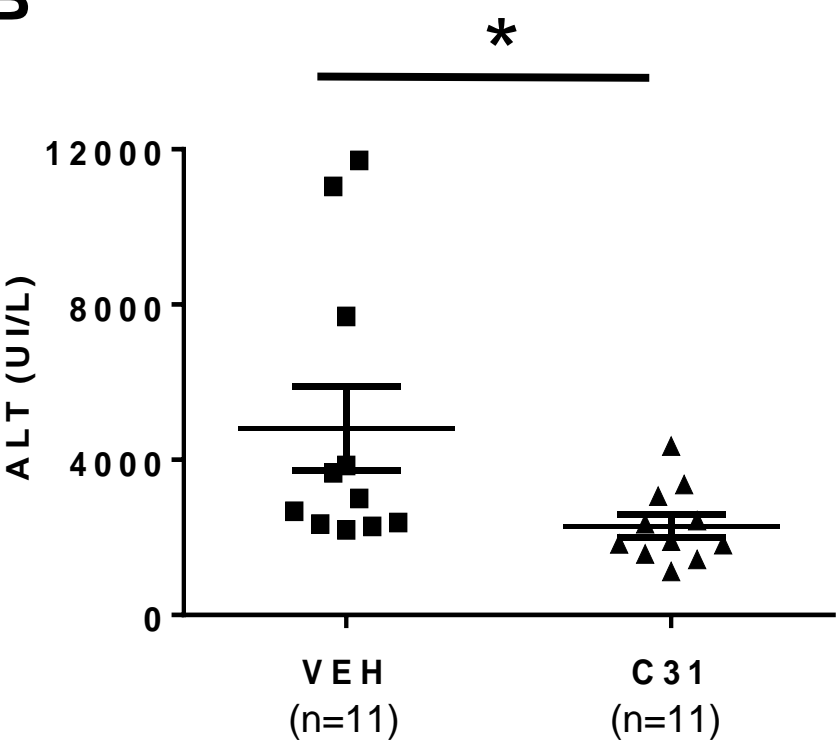

C

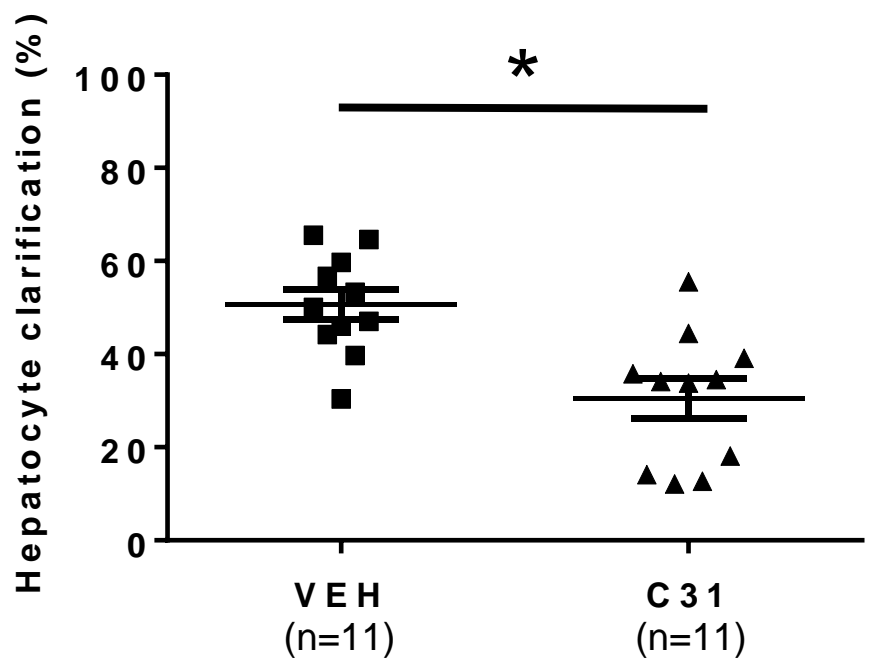

D

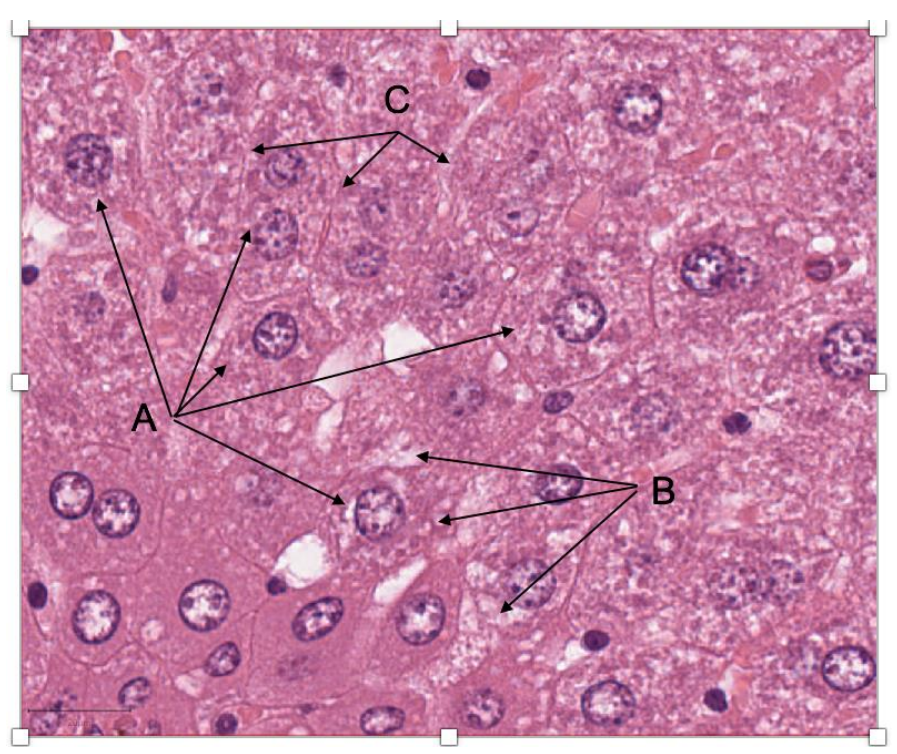


E

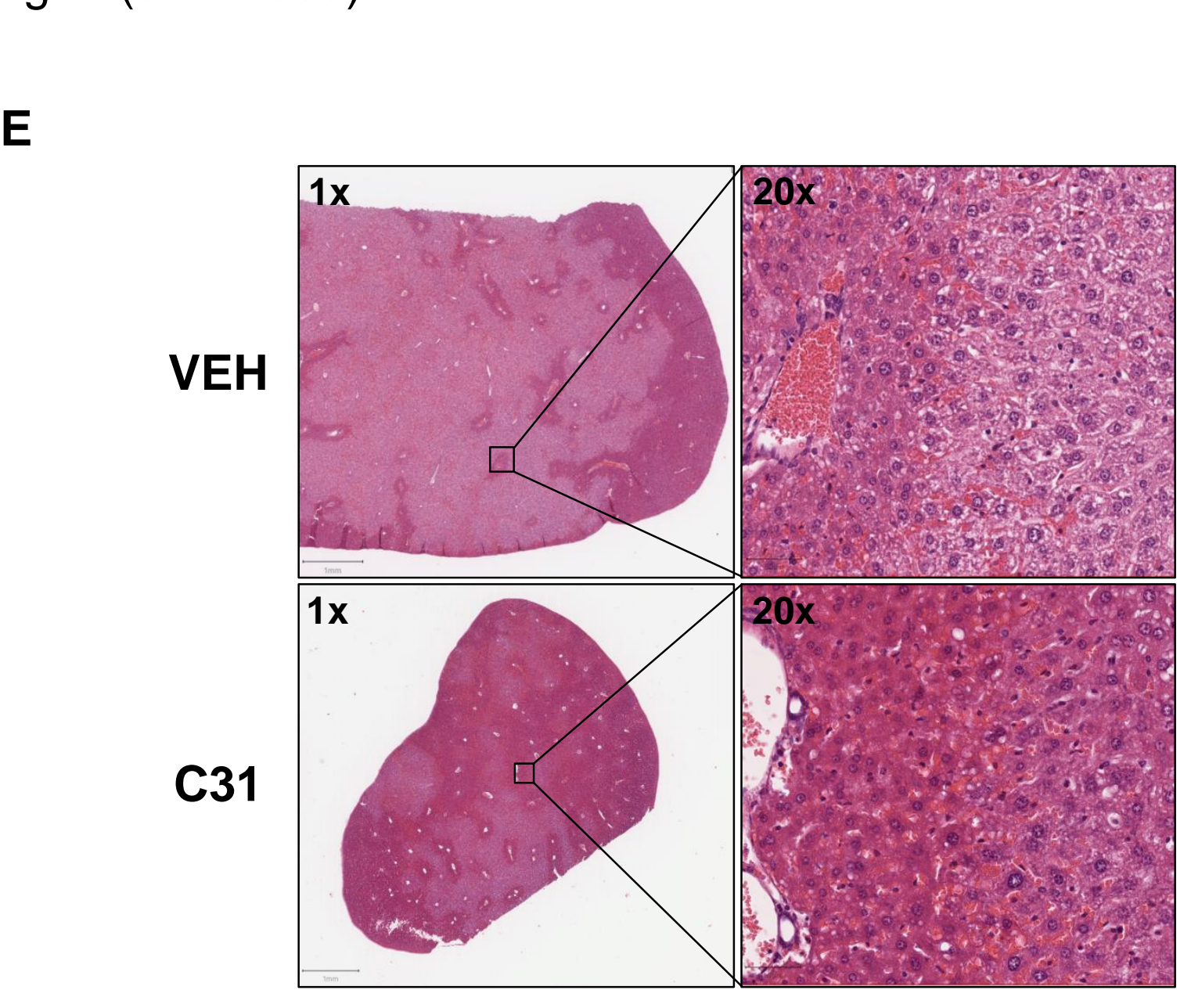

Fig. 7 (continued)

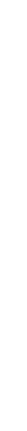

列 